\title{
Circumventing the Dephasing and Depletion Limits of Laser-Wakefield Acceleration
}

\author{
Alexander Debus $\odot,{ }^{1, *}$ Richard Pausch, ${ }^{1,2}$ Axel Huebl, ${ }^{1,2}$ Klaus Steiniger, ${ }^{1,2}$ René Widera, ${ }^{1}$ \\ Thomas E. Cowan, ${ }^{1,2}$ Ulrich Schramm, ${ }^{1,2}$ and Michael Bussmann ${ }^{1}$ \\ ${ }^{1}$ Helmholtz-Zentrum Dresden-Rossendorf (HZDR), Bautzner Landstraße 400, 01328 Dresden, Germany \\ ${ }^{2}$ Technische Universität Dresden, 01062 Dresden, Germany
}

(Received 5 March 2018; revised manuscript received 4 March 2019; published 10 September 2019)

\begin{abstract}
Compact electron accelerators are paramount to next-generation synchrotron light sources and freeelectron lasers, as well as for advanced accelerators at the $\mathrm{TeV}$ energy frontier. Recent progress in laserplasma driven accelerators (LPA) has extended their electron energies to the multi-GeV range and improved beam stability for insertion devices. However, the subluminal group velocity of plasma waves limits the final electron energy that can be achieved in a single LPA accelerator stage, also known as the dephasing limit. Here, we present the first laser-plasma driven electron accelerator concept providing constant acceleration without electrons outrunning the wakefield. The laser driver is provided by an overlap region of two obliquely incident, ultrashort laser pulses with tilted pulse fronts in the line foci of two cylindrical mirrors, aligned to coincide with the trajectory of the accelerated electrons. Such a geometry of laterally coupling the laser into a plasma allows for the overlap region to move with the vacuum speed of light, while the laser fields in the plasma are continuously being replenished by the successive parts of the laser pulses. Our scheme is robust against parasitic self-injection and self-phase modulation as well as drive-laser depletion and defocusing along the accelerated electron beam. It works for a broad range of plasma densities in gas targets. This method opens the way for scaling up electron energies beyond $10 \mathrm{GeV}$, possibly towards $\mathrm{TeV}$-scale electron beams, without the need for multiple laser-accelerator stages.
\end{abstract}

DOI: 10.1103/PhysRevX.9.031044

Subject Areas: Optics, Photonics, Plasma Physics

\section{INTRODUCTION}

Laser-wakefield accelerators (LWFA) [1-13] are driven by ultrashort, intense laser pulses traversing an underdense plasma, thus exciting a charge-density plasma wave. Such a wakefield features high fields with acceleration gradients at several $100 \mathrm{GV} / \mathrm{m}$ [14] -3 orders of magnitude higher than in conventional rf accelerators-thus enabling $\mathrm{GeV}$ electron accelerators on a length scale of centimeters.

Two major limitations of LWFA, the dephasing length and the depletion length, presently prevent reaching even higher electron energies in the $100 \mathrm{GeV}$ to $\mathrm{TeV}$ range. On the one hand, the dephasing length [15]

$$
L_{\mathrm{d}}=4 / 3\left(\omega_{0}^{2} / \omega_{p}^{3}\right) \sqrt{a_{0}} c
$$

is the characteristic length for a relativistic electron to outrun the accelerating region of the wakefield and thus the maximum acceleration distance, with $\omega_{0}$ and $\omega_{p}$ denoting

\footnotetext{
*a.debus@hzdr.de
}

Published by the American Physical Society under the terms of the Creative Commons Attribution 4.0 International license. Further distribution of this work must maintain attribution to the author(s) and the published article's title, journal citation, and DOI. laser frequency and plasma frequency, respectively. The laser strength parameter $a_{0}$ is defined as the peak amplitude of the normalized vector potential of the laser field $e A_{0} / m c^{2}$ and is related to the peak intensity of a linearly polarized laser by $a_{0} \simeq 0.85 \times 10^{-9} \lambda_{0}[\mu \mathrm{m}] I_{0}^{1 / 2}\left[\mathrm{~W} / \mathrm{cm}^{2}\right]$. On the other hand, the laser pump depletion length [15]

$$
L_{\mathrm{dep}}=\left(\omega_{0} / \omega_{p}\right)^{2} c \tau_{0},
$$

with laser pulse duration $\tau_{0}$, denotes the length scale for laser pulse energy depletion in driving the wakefield.

Higher energy gains $\propto n_{e}^{-1}$ in a LWFA can be achieved by lowering the plasma density, thus decreasing plasma frequency $\omega_{p} \propto n_{e}^{1 / 2}$ and increasing both $L_{\mathrm{d}}$ and $L_{\text {dep }}$. However, when keeping the target electron beam energy constant at any suitably low density according to $L_{\mathrm{d}} \propto n_{e}^{-3 / 2}$, this scaling comes at the cost of increasing laser pulse energy and accelerator length $\propto n_{e}^{-1 / 2}$ for the target electron beam energy [15-17] while adding difficulties in maintaining drive-laser guiding over the extended distance $[10,11,13]$.

Despite state-of-the-art ultrashort, petawatt-scale lasers easily exceeding intensity and pulse energy requirements of LWFA self-injection [18], it is this dephasing and depletion limit that currently constrain LWFA peak energies to a range of hundreds of $\mathrm{MeV}$ to several $\mathrm{GeV}[9,11,13]$. 
In principle, these limitations can be overcome by using multiple LWFA stages to successively accelerate one electron beam to higher energies. However, in practice, this approach introduces severe challenges with regard to electron beam transport in between stages [19-23], which need to be spaced apart due to coupling in and coupling out of the laser beam, hence leading to charge loss and emittance growth.

Thus, extending the beam energy gained within a single stage beyond the dephasing limit is a prime objective in the development of plasma-based accelerators: In LWFAs, spatially tapering the plasma density profile within a plasma waveguide can extend $L_{\mathrm{d}}$ by precisely tailored density down-ramps [24,25], which speed up the plasma phase velocity in order to synchronize accelerating electrons with the wakefield phase. Such wakefields can, in principle, be maintained indefinitely at the price of a rapidly decreasing acceleration gradient. Conversely, density up-ramps $[26,27]$ can be utilized for rephasing the accelerating electrons within a spatially shrinking wakefield, until, in the best case, the final acceleration distance reaches the hard limit of $L_{\text {dep }}$ [Eq. (2)].
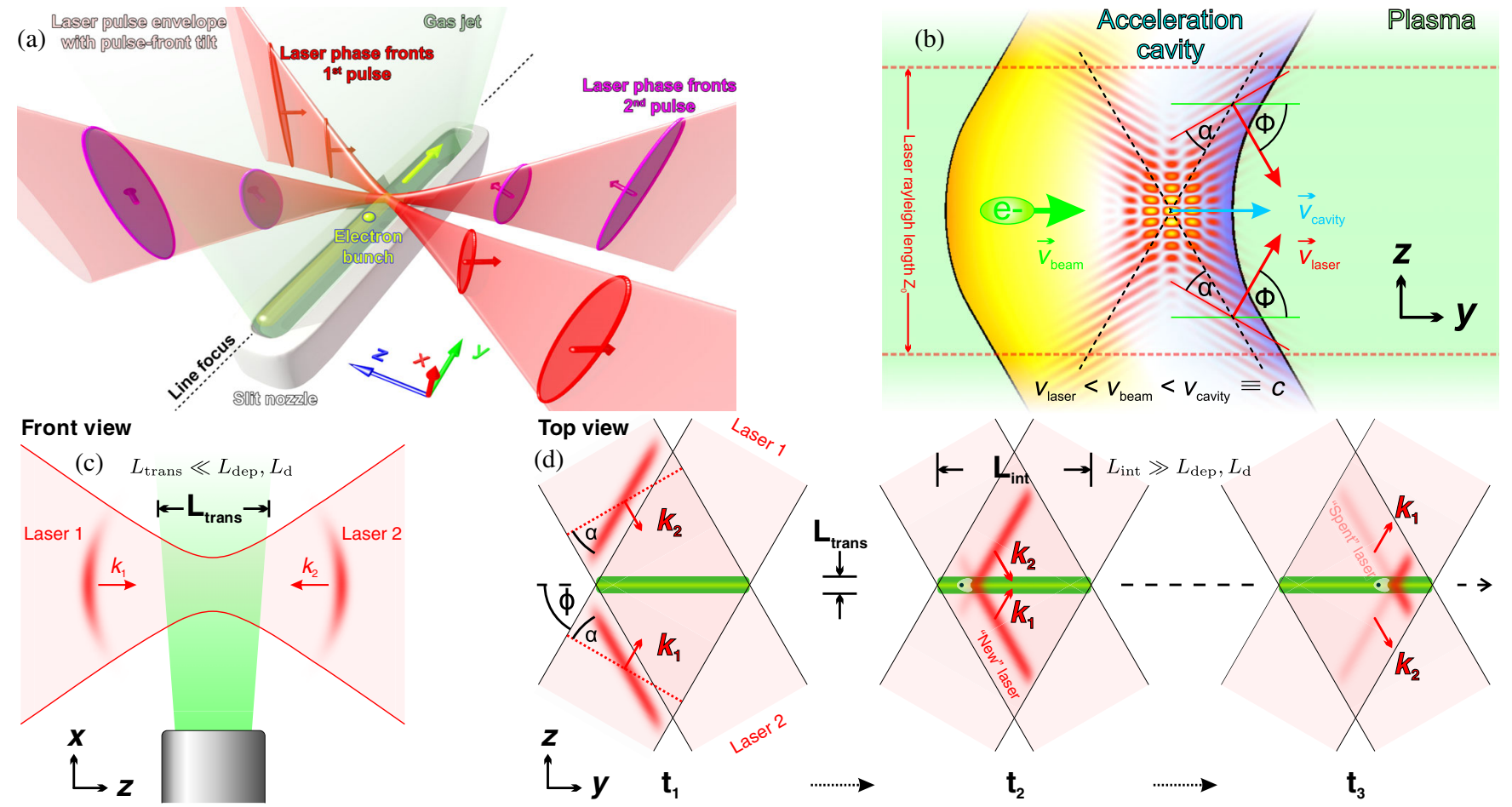

\section{TRAVELING-WAVE ELECTRON ACCELERATION}

We begin with an overview as shown in Fig. 1 in order to subsequently explain how an intersection of two

FIG. 1. TWEAC features a laser-plasma interaction region, which propagates with exactly the speed of light, thus remaining synchronized to the accelerated electron bunch arbitrarily far beyond the dephasing length — limited by the spatial extent of the laser pulse only. (a) Illustration of the required geometry of two ultrashort, pulse-front-tilted, obliquely incident laser beams in a line focus, driving a wakefield in a slit-nozzle generated gas jet for accelerating an electron bunch. Surfaces of equal phase within each laser beam mark the direction of pulse propagation (here, $60^{\circ}$ with respect to the main axis) relative to the pulse-front tilt. (b) Zoomed-in schematic of the accelerating region. The two TWEAC lasers (red intensity contours) drive a copropagating V-shaped plasma cavity with $v_{\text {laser }}<v_{\text {beam }}<v_{\text {cavity }} \equiv c$ that accelerates an electron bunch in its longitudinal cavity field (orange-white-purple). (c) Cylindrical focusing geometry. (d) Illustration of the comoving laser overlap, as well as the laser field cycling through the laser-plasma interaction region. 
pulse-front-tilted laser pulses can be exploited to drive a plasma wakefield cavity for electron acceleration, which, on the one hand, indefinitely remains ahead of the electrons to avert dephasing and, on the other hand, provides a way to continuously replenish the laser-driver field to avert laser pump depletion.

The core guiding principle of our approach, TravelingWave Electron Acceleration (TWEAC), is to create a laser focal region that moves ideally with exactly the vacuum speed of light and thus faster than the plasma group velocity $v_{g}<c$. The first goal is that accelerated electrons cannot overtake the accelerating structure, so that a dephasing limit does not exist in this type of wakefield accelerator, and relativistic electrons remain stationary with respect to a comoving wakefield. The second central aspect of TWEAC is that a stable and experimentally controllable plasma cavity is achieved by having at every instant a new, unspoiled section of the laser pulse, which has not yet undergone self-phase modulation, transversely entering the plasma and, after only a short propagation distance, forming the acceleration cavity in plasma regions previously unperturbed by lasers. Such a mechanism locally eliminates the laser pump depletion limit as defined by Eq. (2) within the comoving interaction zone, leading to stationary plasma dynamics in which the laser pulse properties directly determine the wakefield properties without being increasingly modified by the plasma response. These two features make it possible to arbitrarily extend such a laser-plasma driven accelerator to higher energies by increasing the length of the TWEAC stage-limited only by the available laser pulse energy but not by the dynamics of the accelerating plasma cavity.

As illustrated in Fig. 1, these objectives can be achieved by an intense, regionally confined and spatially linesymmetric focal zone, created by overlapping two obliquely incident ultrashort laser pulses within an underdense plasma in the line foci of two cylindrical mirrors, aligned to coincide with the trajectory of accelerated electrons. The line focus and laser direction of propagation enclose the interaction angle $\phi$. In contrast to LWFA, the focal region is created directly by optics over the entire interaction zone without the need for laser (self-)guiding in plasma. In order for the overlapping region of the two laser pulses to move along the focal line with the speed of light, both pulses need to match a pulse-front tilt of $\alpha=\phi / 2$ [38,39] [Fig. 1(b)]. In an underdense plasma, the ponderomotive force of such a comoving field geometry features line symmetry with respect to the electron beam trajectory and drives a laser wakefield that moves at exactly the speed of light and accelerates an electron bunch [Fig. 1(b)].

This very specific geometry of lasers is similar to the one also required for high-yield, ultrashort Thomson scattering and all-optical free-electron lasers [38-41]. Technical realization of such pulses in high-power lasers will require additional standard grating optics, as well as cylindrical mirrors [42]. In terms of dispersion precompensation, the pulse synthesis in experiment is not expected to exceed the level of technical challenges that are already met by existing laser pulse compression systems of ultrashort, high-power lasers $[38,39]$. Extensive background on the TWEAC pulse synthesis, as well as its technical requirements, can be found in the references mentioned above and the Appendix A.

Note that the setup satisfies the condition of continuously replenishing the laser field in the focal region; thus, laser pulse evolution is determined not by the total duration of laser-plasma interaction but rather by the much shorter timescale each part of the laser beam needs to traverse the focal region. After a typical distance $L_{\text {cyc }}=L_{\text {trans }} / \sin \phi$, with $L_{\text {trans }}=\min \left(Z_{0}, R_{p}\right)$ being determined by the laser Rayleigh length $Z_{0}$ and plasma channel radius $R_{p}$, the entire laser field has, by propagation, cycled through the central laser-plasma interaction region, where it is subject to laser self-phase modulation and depletion. Hence, in a TWEAC accelerator, it is this field-cycling distance $L_{\text {cyc }}$ and not the total accelerator length $L_{\text {int }}$ that needs to be shorter than the corresponding depletion length according to Lu et al. [15] [see Eq. (2)]: $L_{\text {cyc }}<L_{\text {dep }}$.

This reduction in nonlinear laser-plasma interactions enhances the leverage of experimenters for controlling LPA evolution by its initial laser and gas properties separately, and, for constant laser and gas properties, results in quasistationary conditions beyond Eqs. (1) and (2).

Since self-injection from the back of a bubble relies on electrons moving faster than the wakefield structure itself, the luminal speed of the traveling-wave driven wakefield structure prevents this injection mechanism for quasistationary wakefields. Therefore, injection has to be provided by other mechanisms, such as external, ionization, optical, or density-ramp injection [9], which do not rely on longitudinally overtaking the wakefield structure.

\section{PARTICLE-IN-CELL SIMULATIONS OF TWEAC}

We simulate TWEAC using the 3D3V particle-in-cell code PIConGPU [43] (Appendix B) and compare the result to a standard LWFA scenario. At first, we investigate the plasma dynamics of TWEAC and LWFA based on the same plasma density and peak laser intensity. This perspective has the benefit of similar acceleration gradients and plasma cavity dimensions, which enables us to study fundamental accelerator characteristics.

However, the different geometries of TWEAC and LWFA can give rise to different laser pulse energies. Depending on the choice of the TWEAC incidence angle $\phi$, the laser pulse energy required to accelerate to some final electron energy can be either higher or lower than for LWFA. Therefore, an additional comparison of LWFA and TWEAC from a facility and application perspective 
becomes equally important. In a second step, we thus examine TWEAC and LWFA using the same laser pulse energy. Finally, we connect these two perspectives using scaling laws and discuss practical considerations relevant for various accelerator regimes and applications.

For the initial analysis of fundamental plasma dynamics, we choose a plasma with a comparably high electron density of $n_{e}=3.2 \times 10^{18} \mathrm{~cm}^{-3}$ and short laser pulse of $\tau_{0}=10$ fs duration (FWHM), so that both $L_{\mathrm{d}}=5.7 \mathrm{~mm}$ and $L_{\mathrm{dep}}=4.9 \mathrm{~mm}$ are short. Since self-injection is prevented in TWEAC by an interaction region moving at the speed of light, we trigger electron injection by an initial density shock [44-47] followed by a $620-\mu \mathrm{m}$ density downramp. After injection, the simulated plasma length for acceleration $L_{\text {int }} \simeq 12 \mathrm{~mm}$ exceeds twice $L_{\mathrm{d}}$ and $L_{\mathrm{dep}}$.

As discussed in more detail later, both TWEAC-driving laser fields with $800-\mathrm{nm}$ wavelength are incident at $\phi= \pm 60^{\circ}$, and each features a pulse-front tilt of its intensity front of $\mp 30^{\circ}$ with respect to the laser wavefronts. The peak laser field strength on each arm is $a_{0}=3.5$, so the maximum laser strength within the optical lattice in the overlap region reaches $2 \cdot a_{0}$. In order to confine the width of the interaction region along $z$ to several plasma skin depths $k_{p}^{-1}$, we choose a small height of the line focus $w_{0, x}=1.2 \mu \mathrm{m}$. Compared to the matched LWFA $1 / e^{2}$ laser spot size $w_{0}=2 \sqrt{a_{0}} / k_{p}$ for self-guiding [15] of $w_{0}=$ $11 \mu \mathrm{m}$ for $a_{0}=3.5$, we operate in a strongly overfocused regime, so the laser pulse immediately diffracts again after reaching the focus without being self-guided by the plasma cavity it creates. From an experimental point of view, such a thin line focus averts the onset of self-focusing [9,48], which otherwise would unnecessarily drive the plasma where no cavity formation is required and increase $L_{\text {trans }}$ from the $Z_{0}$ scale to, at maximum, the plasma channel radius $R_{p}$. According to $P / P_{c}=\left(k_{p} w_{0} a_{0}\right)^{2} / 32 \leq 1$ [9] and each of the laser pulses at $a_{0}=3.5$, the laser power $P / P_{c}=0.06<1$ remains far below the self-focusing threshold. From a numerical perspective, the small focal size enables us to use a simulation size that transversely extends beyond 18 Rayleigh lengths and thus includes the relevant laser-plasma dynamics.

\section{CAVITY FORMATION IN TWEAC}

The ponderomotively driven plasma dynamics of the TWEAC scenario are similar to LWFA in the $y x$ plane [Figs. 2(a) and 3(a)], where the blowout radius of the plasma cavitation follows the known $R \sim 2 \sqrt{a_{0}} / k_{p}$ relation [15]. However, in the $y z$ plane of laser propagation, we observe a very distinct cavity formation, which arises from the TWEAC geometry. The two incoming arms of the lasers expel electrons along a characteristic V-shaped baseline profile with an opening angle of $\pi-\phi$ and a transverse width comparable to the laser Rayleigh length $Z_{0}$ [Figs. 2(b) and 3(b), right].

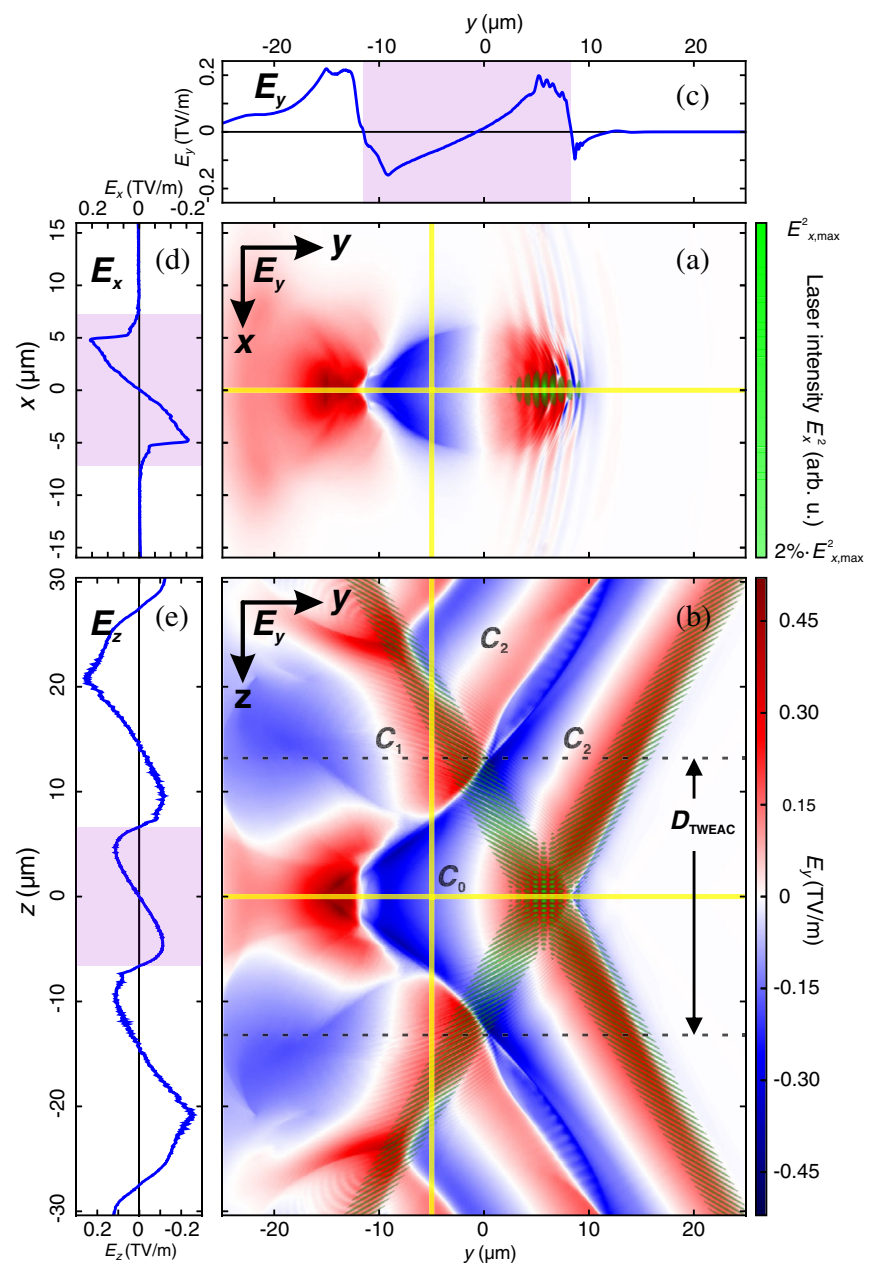

FIG. 2. Traveling-Wave Electron Acceleration fields $E_{y}$ are on the order of several $100 \mathrm{GV} / \mathrm{m}$. (a,b) The longitudinal acceleration $E_{y}$ in (a) the $x y$ plane and (b) the corresponding orthogonal laser plane of propagation $(y z)$. The projections (c)-(e) show the acceleration and transverse focusing fields at selected axes (yellow), respectively. For illustrating the laser pulse position within the cavity, the intensity (green) is shown in the range $[0.02,1.0] \cdot E_{x, \max }^{2}$. Here, $D_{\text {TWEAC }}$ marks the maximum extent of the central cavity $C_{0}$ in the $y z$ plane, and side cavity structures are labeled as $C_{1}$ (behind both lasers) and $C_{2}$ (between the laser arms). Because of the difference in transverse cavity extent defined by the laser pulse geometry, the focusing fields of the $x y$ plane are stronger compared to the $y z$ plane.

Note that in spite of the presence of an optical lattice, the central overlap region of the lasers is effective in ponderomotively evacuating the plasma cavity (Fig. 3). Such a lattice could, in principle, lead to changes to the ponderomotive dynamics [49-51] or stochastic heating [52,53]. Particularly, these dynamics could allow for transmissive modes, where fractions of the electron density in front of the laser transit straight through the laser beam into the cavity behind, thus diminishing cavity fields. However, in a number of test simulations, we established that our TWEAC scenario, based on linearly polarized lasers in $x$, 

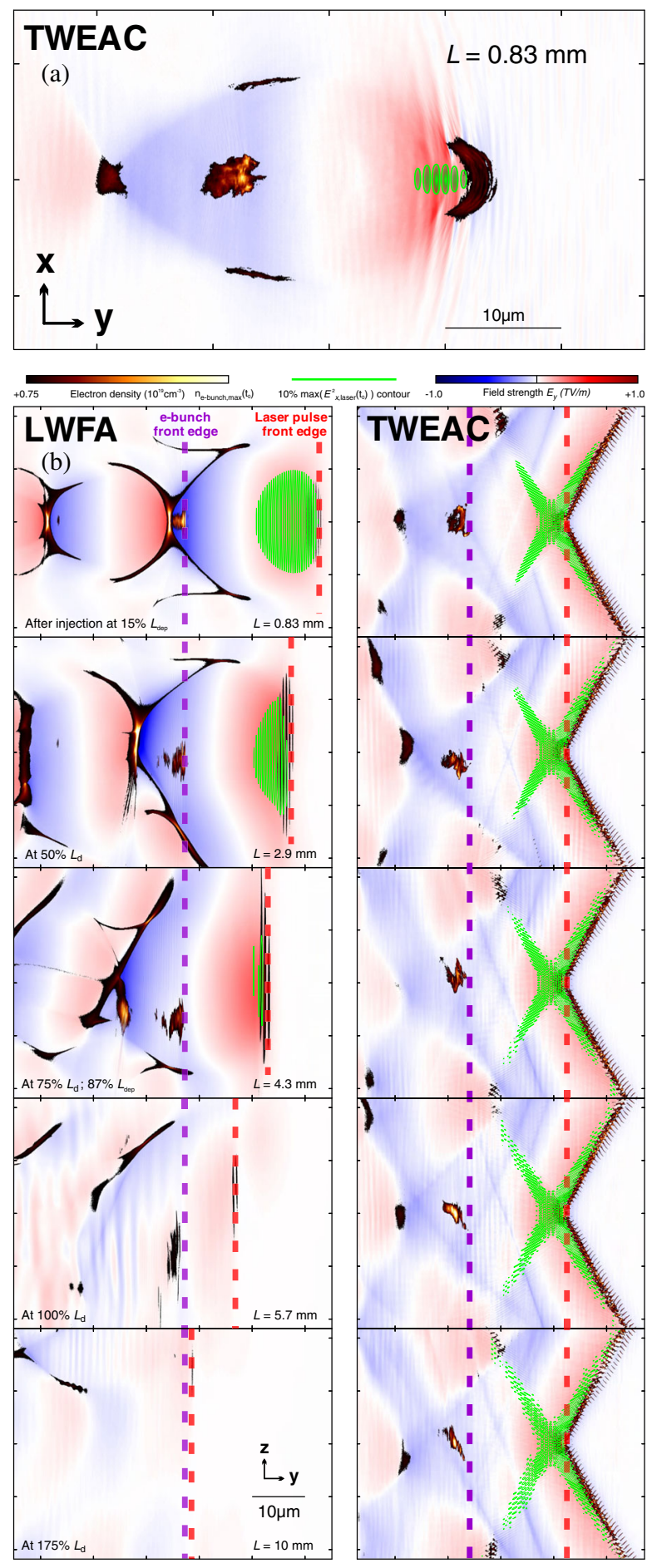

FIG. 3. Comparison of the laser, cavity, and electron bunch evolution of LWFA and TWEAC, at characteristic stages of interaction until the acceleration length $1.75 L_{\text {dep }}$, thus illustrating that quasistationary laser fields of TWEAC lead to continued electron acceleration. (a) The $x y$ plane and (b) the $y z$ plane. Each figure shows the respective accelerating cavity field $E_{y}$, electron density beyond $n_{e}=0.75 \times 10^{19} \mathrm{~cm}^{-3}$, and laser field intensity contour $\propto E_{x}^{2}$ at $10 \%$ of the maximum initial intensity. Two lines to guide the eye mark the front edge of the electron bunch (purple) and the rising edge of the laser pulse (red) during acceleration, such that a decreasing separation highlights drive-laser dephasing. features minimal transmissive modes in a broad range extending from $\phi=30^{\circ}$ to $70^{\circ}$. Within this range, the optical lattice node spacings in each dimension remain comparable to the laser wavelength, so the larger scale intensity variations are determined by the laser envelope and not the lattice structure. Outside this angle range, it is necessary to use two lasers with nonparallel or even elliptical polarization vectors, such as orthogonally orientated linear polarization [later in Fig. 5(a)] or counterrotating circular polarization, while satisfying line symmetry in laser polarizations with respect to the electron beam axis.

The resulting cavity fields of the TWEAC scenario before electron injection (Fig. 2) show that these are comparable to LWFA, in magnitude with respect to accelerating and focusing fields, as well as in linearity with respect to its field gradients. Differences mainly arise due to the particular TWEAC cavity geometry, which is rotationally asymmetric with respect to the accelerating and focusing wakefield and leads to focusing fields $E_{z}$ and $E_{x}$ differing by a factor of 2 . While in the plane normal to laser beam propagation the field geometry [Fig. 2(a)] is the same as in LWFA, the in-plane fields [Fig. 2(b)] feature a V-shaped, cylindrical cavity structure determined by the laser interaction angle $\phi$.

Beyond a central region of overlapping lasers measuring $c \tau_{0} / \sin (\phi / 2)$ in transverse size, the two lasers can only drive a shared plasma cavity if the incoming arm of one laser and the outgoing arm of the other laser are separated by less than one plasma period $2 \pi c / \omega_{p}$ along an axis perpendicular to the incoming laser pulse front. For larger separations, each arm drives its own wake [Fig. 2(b)]. Thus, the characteristic transverse diameter of a TWEAC cavity is

$$
D_{\text {TWEAC }}=\max \left(\frac{\lambda_{p}}{\sin (\phi / 2)}, \frac{c \tau_{0}}{\sin (\phi / 2)}\right) .
$$

For the electron density $6.4 \times 10^{18} \mathrm{~cm}^{-3}$ before injection [Fig. 2(b)], the estimate $D_{\text {TWEAC }}=26.4 \mu \mathrm{m}$ is in excellent agreement with the simulation results. Because of the high laser intensity, separate cavity formation of the respective laser pulses continues beyond the short laser focal depth $Z_{0}=20.1 \mu \mathrm{m}$ and does not start to terminate until $z= \pm 32 \mu \mathrm{m}$.

\section{ELECTRON PROPERTIES}

The comparison of electron spectra in Fig. 4 demonstrates TWEAC to be completely unaffected by the LWFA limits. For the standard LWFA scenario, the electron spectra show significant electron beam degradations, as expected from wakefield dephasing, laser energy depletion, and multiple electron bunch self-injection events [Fig. 4(b)]. After an initial acceleration up to $769 \mathrm{MeV}$ before the dephasing length, the 78-pC electron beam decelerates until 594-MeV mean bunch energy and 

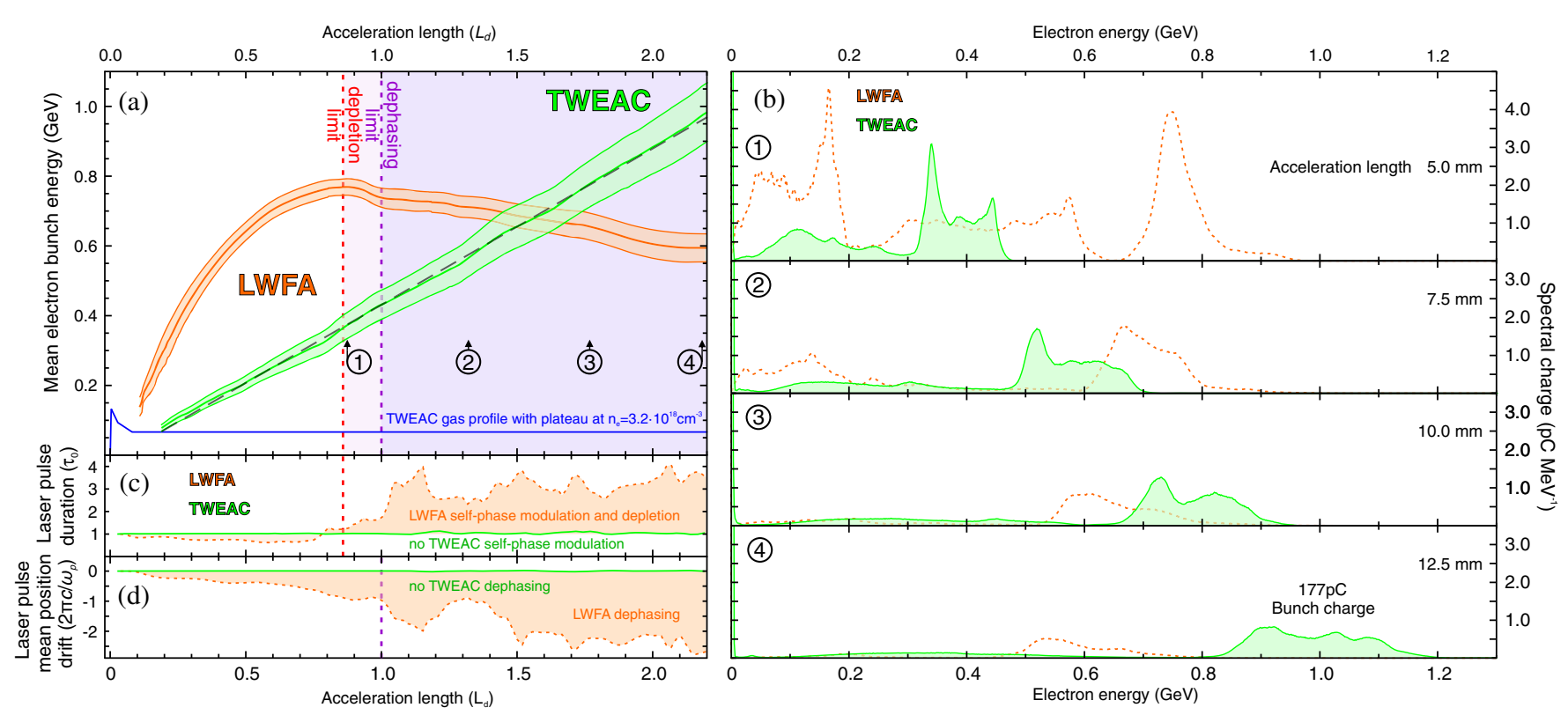

FIG. 4. Comparison of a LWFA and a $60^{\circ}$-TWEAC scenario at the same plasma density and peak laser intensity, contrasting the accelerators based on similar plasma dynamics. (a) The mean electron bunch energy of Traveling-Wave Electron Acceleration (green) and a corresponding self-injected laser-wakefield accelerator (orange) with respect to the accelerated distance normalized to the dephasing length $L_{\mathrm{d}}=5.70 \mathrm{~mm}$. The bunch energy spread (standard deviation) is represented by the shaded areas, respectively. For TWEAC, the acceleration remains constant and does not saturate within the dephasing or depletion length. The linear fit (gray, dashed line) highlights the excellent linearity of the TWEAC acceleration over the entire distance. (b) Electron spectra of all electrons in the TWEAC (green, solid line) and LWFA (orange, dashed line) scenario that are propagating within a 27-mrad (total angle) cone along the $y$ axis at subsequent stages of acceleration. (c) The on-axis pulse duration of the TWEAC scenario compared to a standard LWFA, illustrating the absence of self-phase modulation achieved through the field-cycling mechanism in TWEAC. (d) The respective drift of the average on-axis longitudinal laser position compared to a test particle moving with the speed of light. Within numerical accuracy, the TWEAC laser field remains stationary and thus displays no dephasing.

$13.5 \%$ energy spread (rms) are reached at the end of the simulated distance.

The TWEAC scenario features no such limitations, and electron acceleration progresses strictly linearly with accelerator length up to the $\mathrm{GeV}$ scale at the end of the simulation [see Fig. 4(a)], so the total accelerator length far exceeds both LWFA depletion and dephasing lengths. The electron bunch has a comparably high charge of $177 \mathrm{pC}$ with mean energy and energy spread of $984 \mathrm{MeV}$ and $17.1 \%$ (rms), respectively, surpassing LWFA in electron energy.

Note that despite the absence of self-injection in TWEAC, we still observe parasitic injection into adjacent side cavity structures [Fig. 2(b)] from the initial density ramp, which gives rise to some continuous low-energy background in Fig. 4(b). However, these adjacent cavities $C_{1}$ and $C_{2}$ do not have the strong transversely confining fields of the central TWEAC cavity $C_{0}$; thus, the parasitic electron background is found to continuously diminish in charge over acceleration length, while the total charge within the electron bunch stabilizes after injection and remains constant afterward.

Furthermore, we note that the observed electron beam quality is mainly determined by the density-shock injection used in this specific example, which by tailoring the density down-ramp, we explicitly optimize towards a high bunch charge and not for minimum energy spread. By demonstrating uniform acceleration of such a spatially extended, high-charge electron beam to a high electron energy, rather than a small bunch, confined to a small phase-space volume, we avoid resorting to an edge case and demonstrate useful acceleration gradients both longitudinally and transversely. Consequently, TWEAC is expected to be applicable to a whole range of different initial electron pulses and injection mechanisms [54,55], so the desired beam quantities, such as a low electron energy spread, will improve greatly once injection-optimized regimes are pursued.

Finally, the observed increase in absolute energy spread during acceleration is predominantly caused by numeric effects; see Appendix B 2.

\section{LASER FIELDS ARE QUASISTATIONARY}

For further increases in electron energy far beyond the example shown in this work, it is essential to demonstrate that in contrast to LWFA, drive-laser evolution in TWEAC is indeed quasistationary as shown in Figs. 3, 4(c), and 4(d). While the LWFA laser pulse is completely obliterated, as the field amplitude drops by more than 2 orders of 
magnitude, the driver field in TWEAC remains constant. Likewise, LWFA laser pulse duration more than triples due to self-phase modulation and depletion, where TWEAC laser duration is constant within 3\% [Fig. 4(c)]. An equally stationary result is obtained for drive-laser dephasing [Fig. 4(d)], measured as the mean longitudinal position drift of the laser field compared to a test particle traveling at the speed of light. In LWFA, it increases to beyond 2.5 plasma periods, whereas TWEAC dephasing less than $4.0 \times$ $10^{-3}\left(2 \pi c / \omega_{p}\right)$ is nonexistent within numerical precision. The remaining variations in laser properties most likely have to be attributed to accumulated numerical errors over the simulation duration of $500 \times 10^{3}$ time steps, thus showing that effects from self-phase modulation and other instabilities are indeed negligible. In principle, bunch-internal or bunchcavity particle dynamics, such as from betatron oscillations, lead to bunch slippage with respect to the cavity and hence present an additional source of dephasing. However, in the simulated scenario, the mean electron bunch position slips by less than $0.5 \mu \mathrm{m}$ over a total acceleration length of $12.5 \mathrm{~mm}$. Both Figs. 4(c) and 4(d) show good agreement with the respective analytical estimates of the depletion, Eq. (2), and dephasing limit, Eq. (1).

Specifically, Figs. 4(c) and 4(d) show that the dephasing and depletion limits are not only barely overcome, but up to numerical accuracy, they do not apply at all, even after more than twice $L_{\mathrm{d}}$ and $L_{\mathrm{dep}}$. This excellent stability of the drive laser over time combined with the ability of the resulting plasma cavity to trap and accelerate electrons clearly displays the potential of TWEAC to scale up to energies that are by several orders of magnitude beyond the $\mathrm{GeV}$ scale shown in our TWEAC simulation. Stated in other terms, the stationary dynamics of constant acceleration phase and gradient makes TWEAC functionally a laser-plasma analog of the Traveling-Wave Linac structures used in conventional rf-based linacs [56,57]. Simulating even longer TWEAC setups using current state-of-the-art 3D-PIC codes becomes increasingly numerically demanding; see Appendix B 2.

\section{LASER PULSE ENERGY SCALING}

So far (Figs. 2-4), we have studied the fundamental plasma dynamics of TWEAC and LWFA while neglecting the energy efficiency of the new acceleration scheme, which beyond proof-of-principle realizations, is decisive for the practicability of TWEAC for applications. In the following, we examine the respective laser pulse energies required and the resulting scaling properties.

For achieving a high $a_{0}$ over an interaction length $L_{\text {int }}$, the total required laser pulse energy for both TWEAC lasers is

$$
W_{0}=\frac{\pi c^{3} m_{e}^{2} \varepsilon_{0} \omega_{0}^{2}}{2 q_{e}^{2} \sqrt{2 \log 2}} a_{0}^{2} \tau_{0} L_{\mathrm{int}} w_{x} \sin (\phi)
$$

Towards longer accelerator lengths $L_{\mathrm{int}}$, and thus higher electron energies $E_{e}$, the required laser pulse energy $W_{0}$ scales linearly with the final electron energy-limited only by the total available laser energy.

For the $60^{\circ}$ scenario, studied until now, Eq. (4) results in $92 \mathrm{~J}$ for 2.6 times the LWFA depletion length compared to $3.3 \mathrm{~J}$ and one depletion length for the LWFA scenario. This comparison immediately shows that the $60^{\circ}$-TWEAC scenario lacks efficiency with respect to required laser energy. Fortunately, the incidence angle $\phi$ is still a free tuning parameter, which according to Eq. (4), scales favorably towards smaller angles, thus enabling high energy efficiencies for TWEAC. That scaling exploits the increased interaction length when a given laser pulse width is on-axis projected using a reduced interaction angle $\phi$ and can be extended to smaller angles $\phi$, as long as the characteristic field-cycling distance remains below the depletion length $L_{\text {cyc }}<L_{\text {dep }}$.

In a further PIC simulation [Fig. 5(a)], repeating the previous scenario with an incident angle of $5^{\circ}$ instead of $60^{\circ}$, the resulting cavity shape resembles the usual LWFA shape, while all advantages of TWEAC are retained. In particular, the simulation has shown similar electron acceleration gradients (106\%) compared to the $60^{\circ}$ scenario. However, while cavity fields are of the same magnitude, only $9.2 \mathrm{~J}$ are required in total laser pulse energy for the entire acceleration distance of $12.6 \mathrm{~mm}$, i.e., $3.6 \mathrm{~J} / L_{\text {dep }}$. This value is an order of magnitude less in laser pulse energy than the total $92 \mathrm{~J}$ required for the $60^{\circ}$-TWEAC scenario and close to the $3.3 \mathrm{~J} / L_{\text {dep }}$ required for the LWFA scenario with $4.9-\mathrm{mm}$ acceleration distance until depletion [Fig. 5(b)]. Hence, this demonstrates similar laser energy efficiency for TWEAC compared to LWFA. In Appendix D, we present a propagation simulation of a $5^{\circ}$ TWEAC-type pulse and confirm that laser energy depletion or filamentation along $L_{\text {cyc }}$ is negligible $(<5 \%)$ in $\mathrm{mm}$-scale transversally extended gas jets that are typical in experiments.

Extending the scaling of TWEAC to higher electron energies and different electron densities [Fig. 5(c)] follows the same general trend as for LWFA [17], such that for a given target electron energy, the required laser pulse energy $W_{0} \propto E_{e} n_{e}^{-1 / 2}$. TWEAC and LWFA both rely on the laser ponderomotive force and thus ultimately can only achieve the same energy efficiency, as defined by laser pulse energy per electron energy gain and number of accelerated electrons. Hence, for a useful comparison of the technological potential of TWEAC and LWFA, in Fig. 5(b), we only select TWEAC scenarios by varying $\phi, w_{0, x}$, or $\tau_{0}$, which match the required laser pulse energy per LWFA depletion length of the $n_{e}$ scaling discussed in Ref. [17].

According to Refs. [58-60], the LWFA optimal beamloading charge scales $Q_{s} \propto n_{e}^{-1 / 2} \cdot a_{0}^{3 / 2}$. In Fig. 5(c), a constant $a_{0}=1.5$ is assumed, such that the optimal beamloading charge at low densities $10^{17} \mathrm{~cm}^{-3}$ is $440 \mathrm{pC}$ 

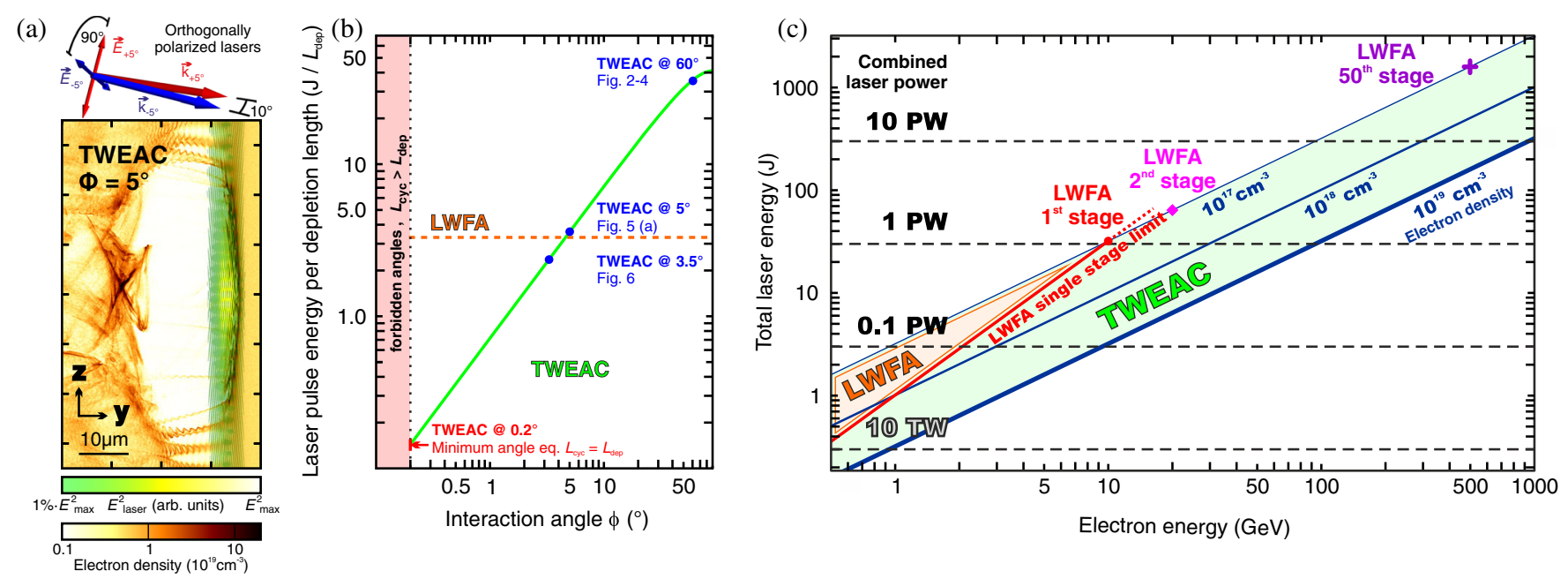

FIG. 5. (a) The TWEAC cavity at shock injection using laser pulses at $5^{\circ}$ incidence angle, both linearly polarized, but orthogonally oriented with respect to each other in order to avoid transmissive plasma modes through standing waves in the laser field. All other initial conditions are kept the same as in Fig. 4. (b) The characteristic wakefield-driver energy of TWEAC [green line, $W_{\text {laser }} / L_{\text {dep }}$ according to Eqs. (2) and (4)] as well as LWFA (orange, dashed line), with the blue markers referring to the respective TWEAC simulations. Denoting the characteristic laser energy required for sustaining a wakefield, $W_{\text {laser }} / L_{\text {dep }}$ is a useful proxy for the total energy efficiency (see Appendix C). The $5^{\circ}$-TWEAC scenario thus demonstrates that these small incidence angles enable electron acceleration at comparable energy efficiency (total laser pulse energy per LWFA depletion length) as LWFA-3.6J $/ L_{\text {dep }}$ (TWEAC) compared to $3.3 \mathrm{~J} / L_{\text {dep }}$ (LWFA). The minimum possible interaction angle $\phi$ is reached when the field-cycling distance equals the corresponding depletion length $L_{\text {cyc }}=L_{\text {dep }}$ (red marker). (c) The total laser pulse energy to electron energy scaling of LWFA and TWEAC at $a_{0}=1.5$ for different electron densities, and the drive-laser energy summed over all acceleration stages, respectively. The corresponding combined laser power requirements (black, dashed lines) assume 30-fs-type laser systems. In contrast to a single-stage TWEAC, markers indicating LWFA setups comprising 1, 2, and 50 stages are shown for $1 \times 10^{17} \mathrm{~cm}^{-3}$ electron density according to Ref. [17]. This scaling illustrates that TWEAC opens up new regimes and has the potential of reaching $\mathrm{TeV}$ energies.

compared to $44 \mathrm{pC}$ at high densities, $10^{19} \mathrm{~cm}^{-3}$. A similar beam-loading scaling is expected to hold also for TWEAC.

The above example illustrates a general trade-off between the accelerated electron bunch charge $Q_{s}$ and the single electron energy gain $E_{e}$ defining the total beam energy $W_{\text {beam }}=\left|Q_{s} / e\right| \cdot E_{e}$, which can only be supplied by the available laser energy $W_{\text {beam }} \leq W_{0}$.

Because of the beam-loading scaling with electron plasma density $Q_{s} \propto n_{e}^{-1 / 2}$, any laser-plasma accelerator at some given total laser energy can either accelerate a high bunch charge at low $n_{e}$ to a low electron energy or a low bunch charge at high $n_{e}$ to a high electron energy. According to Fig. 5(c), a single-staged LWFA at $10^{17} \mathrm{~cm}^{-3}$ can accelerate $440 \mathrm{pC}$ to $10 \mathrm{GeV}$, while a single TWEAC stage of the same laser pulse energy at $10^{19} \mathrm{~cm}^{-3}$ can be exploited to accelerate $44 \mathrm{pC}$ to $100 \mathrm{GeV}$. However, by virtue of not requiring laser pulse guiding structures, TWEAC can operate at much lower electron densities than LWFA, while high-density regimes are accessible because TWEAC removes the density constraints on the acceleration length. This case allows TWEAC the flexibility to exploit a wider range within the bunch charge versus electron energy trade-off than LWFA. In the end, the optimum electron density for a laser-plasma accelerator will depend on the bunch charge and beam quality provided by the electron injection mechanism, as well as the application.
Apart from the general trade-off outlined above, the details of TWEAC beam loading also depend on its different cavity geometry, as well as the polarization state of the two drive-laser beams. As examining this question is beyond the scope of this work, we refer the reader to Appendix $\mathrm{C}$ in which we show a $3.5^{\circ}$-TWEAC scenario that can accelerate electron bunch charges at high energy efficiency [Fig. 5(b) and Table I], significantly in excess of the optimal LWFA beam-loading limit, thus providing a first indication on both robustness and limits of TravelingWave Electron Acceleration at high charge loads.

The primary difference is that TWEAC operates at a single stage level where LWFA requires many subsequent stages. For comparison, markers for 1, 2, and 50 LWFA stages in Fig. 5(c) indicate the high number of stages hypothetically required to reach electron energies beyond $100 \mathrm{GeV}$. Thus, TWEAC immediately eliminates the problem of interstage electron beam transport, as well as laser in-coupling and synchronization issues. This case enables access to new regimes [Fig. 5(c)]: With LWFA on the one hand, $10-\mathrm{GeV}$ electrons from a single stage are in the range of a 1-PW-laser system [17]. With TWEAC on the other hand, a 1-PW-laser system has the potential to reach the $100-\mathrm{GeV}$ energy level within the same accelerator length in a single stage using higher electron densities. Since reaching $1-\mathrm{GeV}$ electron energies becomes possible 
TABLE I. Comparison of various performance and energy efficiency indicators of all LWFA and TWEAC simulations presented. Note that $L_{\text {dep }}$ is calculated for the LWFA laser pulse length $\tau_{0}=30 \mathrm{fs}$ at the respective electron density.

\begin{tabular}{|c|c|c|c|c|}
\hline \multicolumn{5}{|l|}{ Accelerator configuration } \\
\hline Accelerator type & LWFA & TWEAC & TWEAC & TWEAC \\
\hline Incident angle $\phi$ & $\ldots$ & $60^{\circ}$ & $5.0^{\circ}$ & $3.5^{\circ}$ \\
\hline Electron density $\left(\mathrm{cm}^{-3}\right)$ & $3.2 \times 10^{18}$ & $3.2 \times 10^{18}$ & $3.2 \times 10^{18}$ & $2.5 \times 10^{18 \mathrm{a}}$ \\
\hline Peak $a_{0}$ & 7.0 & 7.0 & 7.0 & $6.0^{\mathrm{a}}$ \\
\hline Laser spot size $w_{0, x}(\mu \mathrm{m})$ & 7.9 & 1.2 & 1.2 & 1.2 \\
\hline Polarization state of drive laser & Linearly & Parallel & Crossed & Crossed \\
\hline \multicolumn{5}{|l|}{ Accelerator performance } \\
\hline Characteristic wakefield-driver energy $\left(\mathrm{J} / L_{\mathrm{dep}}\right)$ & 3.28 & 35.60 & 3.58 & 2.36 \\
\hline Cavity volume ${ }^{\mathrm{b}}\left(\mu \mathrm{m}^{3}\right)$ & $7.90 \times 10^{3}$ & $12.8 \times 10^{3 \mathrm{c}}$ & $7.20 \times 10^{3}$ & $30.9 \times 10^{3}$ \\
\hline Mean acceleration gradient $(\mathrm{GeV} / \mathrm{cm})$ & 1.44 & 0.752 & 0.799 & 0.995 \\
\hline Injected charge $(\mathrm{nC})$ & 0.078 & 0.177 & 2.52 & 1.77 \\
\hline Energy-gain efficiency $(\mathrm{J} / \mathrm{GeV})$ & 4.65 & 96.6 & 9.15 & 3.78 \\
\hline Laser-electron beam-energy efficiency $(\%)$ & 1.68 & 0.183 & 27.6 & 46.8 \\
\hline
\end{tabular}

${ }^{\mathrm{a}}$ Reduced plasma density and laser intensity in order to decrease the amount of injected charge.

b Typical cavity volume according to FWHM of background electron density for the first cavity "bucket," respectively.

${ }^{\mathrm{c}}$ The volume determined for the V-shaped cavity of the $60^{\circ}$-TWEAC scenario was limited in the $z$ direction by the size of the simulated gas target.

already for 10-TW-scale laser systems, TWEAC could also be useful for high-repetition-rate applications in the $\mathrm{kHz}$ range [61].

When comparing TWEAC to LWFA schemes comprised of multiple 10-GeV stages, note that each of these LWFA stages supposes the guiding of a PW-class laser in guiding structures, such as discharge capillaries with possible additional heater lasers for providing a suitable density profile $[11,13,62]$. However, when working at high repetition rates, such guiding structures fail today to operate in a stable and reliable way. Particularly, the heating dissipation in such guiding elements may be a severe limitation at high repetition rates. By contrast, TWEAC, with its line-focus geometry, does not require any laser guiding structures and thus is easier to scale in density and spatial dimension, as well as repetition rate.

In addition, the other aspects of multistaged LWFAsuch as the coupling of electron beams and laser pulses, synchronization at the fs level, and the preservation of beam quality, charge, and energy efficiency - are complex and far from being solved. Hence, the ability of TWEAC to drastically reduce if not completely eliminate the need for multiple stages and its beam transport challenges make this scheme a candidate for higher electron energies: On a longer-term perspective, $\mathrm{TeV}$ energies using LWFA currently far exceed existing or near-future laser capabilitieseven if 100 subsequent LWFA stages can be realized at $10^{17} \mathrm{~cm}^{-3}$ densities [Fig. 5(c)]. In contrast, TravelingWave Electron Acceleration, combined with upcoming $10-\mathrm{PW}$-scale laser systems currently under construction [63], has the potential of accelerating at $10^{19} \mathrm{~cm}^{-3}$ density electron bunches with tens of $\mathrm{pC}$ charge to the energy frontier at $\mathrm{TeV}$ energies.

\section{CONCLUSION AND OUTLOOK}

In summary, the novel TWEAC approach mitigates, for the first time, two major limitations of laser-driven plasma accelerators: the dephasing and the depletion limit. Thus, a single acceleration stage can, in principle, be indefinitely extended in length. This scalability is limited only by the available laser pulse energy, as well as the technical capability to sustain the continuous laser beam overlap over an extended accelerator length. Beam transport challenges are minimized because the required gap in between stages for laser pulse in- and out-coupling is significantly reduced. In contrast to LWFA, the obliquely incident laser beams in TWEAC enable stages that can longitudinally be positioned within millimeters next to injectors, subsequent insertion devices, e.g., optical undulators [38,39], or additional accelerator stages. Towards the $\mathrm{TeV}$ energy frontier, TWEAC thus drastically reduces the need for multiple shorter stages $[17,34,35]$ with its associated challenges of interstage beam transport and matching.

From a facility perspective, TWEAC can be customized to a broad range in plasma electron densities and accelerator designs. While the lack of constraints from LWFA dephasing and depletion is advantageous, especially at high densities, TWEAC benefits at low electron densities from not being restricted by laser pulse guiding requirements. As opposed to existing LWFA efforts, it also enables long accelerator stages at electron densities well below $10^{17} \mathrm{~cm}^{-3}$. This ability to match the length of a TWEAC stage to the available laser pulse energies of a laser facility without being constrained in electron density is especially useful for designing high-electron-energy accelerators at high repetition rates. Even if several TWEAC stages are mandated by practical constraints of existing laser facilities, 
such as the maximum laser pulse energy per drive laser, both the acceleration gradient and accelerable charge can be application tailored by the electron density, while interstage beam transport is minimized.

In addition, TWEAC provides unique control of laserdriven plasma accelerators: The resulting plasma cavity propagating faster than the plasma group velocity at exactly the vacuum speed of light effectively eliminates parasitic self-injection, and the continuously renewed laser fields of sideways incident laser beams prevent excessive self-phase modulation of the field driving the wakefield. In the near term, this will improve LPA electron quality for brilliant radiation sources from extreme ultraviolet to the hard $\mathrm{x}$-ray range.

On the middle to long run, these results open up a way to accelerate electrons to the $\mathrm{TeV}$ scale. A stable acceleration gradient of $1 \mathrm{GeV} / \mathrm{cm}$ without dephasing and a continuously renewed laser driver, maintained over $10 \mathrm{~m}$, would provide such a $\mathrm{TeV}$ accelerator.

\section{ACKNOWLEDGMENTS}

The computations were performed on a Bull Cluster at the Center for Information Services and High-Performance Computing (ZIH) at TU Dresden and on the Piz Daint Cluster of the Swiss National Supercomputing Centre (CSCS). We thank the ZIH for its support and generous allocations of computer time. We acknowledge the support of CSCS via Allocation Project No. s795. This project has received funding from the European Unions Horizon 2020 Research and Innovation Programme under Grant Agreement No. 654220.

\section{APPENDIX A: SYNTHESIS OF LASER PULSES WITH TILTED PULSE FRONTS}

Experimentally, TWEAC pulse synthesis differs from traveling-wave Thomson scattering (TWTS) [38-41] mainly in its target application and its more relaxed laser pulse requirements. Although pulse-front tilts can be easily provided by optical gratings, the main challenge is in precompensating for propagation-induced group-delay dispersion (i.e., laser pulse lengthening) due to angular dispersion, which in this case is equivalent to the existence of a pulse-front tilt. This precompensation has to cover the propagation distance starting from the introduction of the tilt angle until the interaction zone, whereby it is essential to provide a sufficiently good match between the central TWEAC axis of electron acceleration and the plane of ideal pulse compression of the optical setup. Especially in Refs. [38,42], the authors show how this can be achieved by using existing ultrashort, high-power lasers by adding two standard optical gratings to the laser beam line after the final optical pulse compressor. For first proof-of-principle experiments using $\mathrm{cm}$-scale TWEAC stages at $\phi<10^{\circ}$, required dispersion precompensation is expected to be minimal to nonexistent.

Providing narrow line foci with $\mu \mathrm{m}$-scale radii is another technical challenge for the spatial beam quality of ultrashort, high-intensity, high-power laser facilities, requiring a suitably high Strehl ratio. Current state-of-the-art laser systems produce laser beam qualities with Strehl ratios in excess of 90\% [64], which enable focal spot size and quality close to the diffraction limit [65].

\section{TWEAC robustness to laser pointing variations}

Laser pointing variations show up in TWEAC as deviations in $\phi$ and $\alpha$ that can, in principle, lead to an overlapping region that is moving with a velocity not equal to the vacuum speed of light, thus giving rise to geometrical beam dephasing. In the following, we show that for typical laser pointing variations of modern CPA laser systems, this effect remains negligibly small.

According to the general matching condition [39]

$$
\alpha(\phi)=\arctan \frac{1-\beta_{0} \cos \phi}{\beta_{0} \sin \phi} \stackrel{\beta_{0}=1}{\simeq} \phi / 2
$$

for the pulse-front tilt $\alpha$, one finds the geometrical slippage impact of nonideal $\phi$ and $\alpha$ from variations in laser pointing by calculating $\left.\eta_{s} \equiv\left(\partial \beta_{0}(\phi+\delta \phi, \alpha(\phi, \delta \phi)) / \partial \delta \phi\right)\right|_{\delta \phi=0} \cdot \delta \phi$. The absolute laser-electron beam slippage distance after an acceleration length $L$ being $d_{s}=\eta_{s} \cdot L$, the maximum allowable acceleration distance for TWEAC is $L_{\max }<\lambda_{p} / 2 \eta_{s}$.

The variations in $\phi$ and $\alpha(\phi)$ depend on the optical setup for introducing the pulse-front tilts. In Ref. [42], we examine this very question for TWTS-based optical FELs, which rely on the same kind of laser pulses but have to meet stricter tolerances in experimental design. In its full generality, the technical details with respect to actual optical setups can be rather involved. However, by assuming a pulse-front-tilt synthesis, based on two-grating setups using standard gratings in near Littrow-angle configurations for good optical efficiency, while using incidence and outgoing angles smaller than $60^{\circ}$, several useful assumptions can be made: First, the pointing variations $\delta \phi$ are very similar in magnitude to the initial variations before the pulse-synthesis setup. Second, resulting variations in pulsefront tilt are negligible compared to the pointing variation, $\delta \alpha \ll \delta \phi$. Then, the TWEAC dephasing length due to laser pointing reads

$$
L_{\max }<\frac{\lambda_{p}}{2 \tan \phi / 2 \delta \phi} .
$$

For the $5^{\circ}$-TWEAC scenario at $3.2 \times 10^{18} \mathrm{~cm}^{-3}$ electron density and a laser pointing variation of $\delta \phi \leq 10 \mu \mathrm{rad}$, which is already required for optimally operating the laser pulse compressors in existing ultrashort lasers, the typical maximum acceleration length of a TWEAC stage before acceleration stops due to laser-pointing-induced slippage is $L_{\max }=21.4 \mathrm{~m}$. 
At larger grating incidence angles compared to $60^{\circ}$, the effective pointing variation $\delta \phi$ can increase, such that the subsequent variation in pulse-front tilt $\delta \alpha(\delta \phi)$ cannot be neglected anymore. Often such corrections are within a factor of 2 in $\eta_{s}$; however, if setups are optimized for passive stabilization by satisfying $\delta \alpha \approx \delta \phi / 2$, one can exploit such a setup geometry to achieve significant increases in $L_{\max }$. Ideally, the condition $\delta \alpha=\delta \phi / 2$ results in $\eta_{s}=0$ and $L_{\max } \rightarrow+\infty$, but imperfect optical setups and laser beams lead to large but finite $L_{\max }$.

If one is to exceed the above limit, another option would be to build several TWEAC stages and exploit the fact that, compared to LWFA, each stage is much longer and interstage distances can be virtually eliminated because laser beams are coupled in and out sideways in contrast to onaxis coupling.

\section{Plasma-density independence of $\alpha$ in translationally symmetric media}

Here, we emphasize that the condition of the overlapping region to move along the focal line at the vacuum speed of light $\alpha=\phi / 2$ is independent of the respective plasma density, despite the plasma index of refraction minimally modifying both direction and group velocity of the laser pulses.

The independence of $\alpha$ from plasma density can be shown by applying a translational symmetry argument as shown in Fig. 6: Assume that some section of the laser beam arrives at a point of incidence $A_{0}$ and time $t_{A, 0}$ at the vacuum-plasma boundary with incident angle $\phi$. In vacuum, the beam would continue to propagate and intersect the focal plane at point $A_{1}$ and time $t_{A, 1}$.

According to the plasma density, the laser beam is, even if only very slightly, refracted to an incident angle $\phi^{\prime}$, and it propagates at a group velocity $v_{g}<c$. After some optical path length, the beam intersects the focal plane of the line focus at point $A_{2}$, which is reached at some time $t_{A, 2}>t_{A, 1}$.

Now, one considers a second point of incidence $B$ of the same pulse-front-tilted laser at the vacuum-plasma boundary at a later time $t_{1}$, where $\overline{A_{0} B_{0}}=c \cdot\left(t_{B, 0}-t_{A, 0}\right)$ due to the laser pulse-front tilt $\phi / 2$ and laser group velocity $v_{g}=c$ in vacuum. This second section of the pulse-fronttilted laser beam continues to propagate in the same direction as the first beam section at point $A$.

Since the density profile within the TWEAC gas jet is translationally symmetric along the laser line focus direction $\left[y\right.$ axis in Fig. 1(d)], the resulting optical path $\overline{B_{0} B_{2}}$ from the vacuum-plasma boundary to the focal plane of the line focus is the same as the optical path $\overline{A_{0} A_{2}}$. Accordingly, the relative timings $t_{B, 2}-t_{B, 0}$ and $t_{A, 2}-t_{A, 0}$ are also the same. From this and $\overline{A_{0} B_{0}}=c \cdot\left(t_{B, 0}-t_{A, 0}\right)$, it follows that the corresponding relative timing between $A_{2}$ and $B_{2}$ also has to be the same as between $A_{0}$ and $B_{0}$.

Therefore, the pulse-front tilt changes according to beam refraction arising from the subluminal plasma group velocity

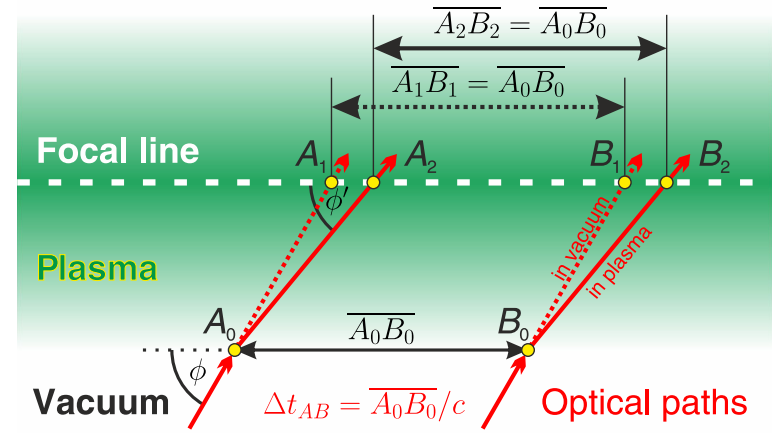

FIG. 6. Laser beam refracted at the vacuum-plasma boundary. Because of the same optical path of $\overline{A_{0} A_{2}}$ and $\overline{B_{0} B_{2}}$ seen by the TWEAC laser at different positions of incidence along the $y$ axis, the difference in arrival times at $\overline{A_{2} B_{2}}$ is the same as $\overline{A_{0} B_{0}}$. Thus, the relation between pulse-front tilt and incident angle $\phi$ does not change in the plasma of a slit-nozzle gas jet, as long as it is translationally symmetric along the line focus.

in such a way that the vacuum speed-of-light propagation of the laser beam overlap region along the laser line focus does not change. Although the plasma changes the absolute laser arrival time at the focal line, the speed of the TWEAC laser overlap remains the same. This result is a direct consequence of the translational symmetry of the TWEAC setup.

\section{APPENDIX B: PARTICLE-IN-CELL SIMULATIONS OF TWEAC}

\section{Simulation model}

We model Traveling-Wave Electron Acceleration using the 3D3V particle-in-cell code PIConGPU $[43,66]$ based on the beta-rc6, 0.3.1, and 0.4.0 releases [67]. The TWEAC laser pulses implement Eq. (6) of Ref. [38] including the dispersion effects due to the pulse-front tilt for ultrashort laser pulses. The simulation volume $(x, y, z)$ of both TWEAC scenarios comprises $880 \times 2304 \times 2784$ cells with cell sizes $\left(\lambda_{0} / 12.0\right) \times\left(\lambda_{0} / 20.9\right) \times\left(\lambda_{0} / 20.9\right)$ for $\lambda_{0}=0.8 \mu \mathrm{m}$, yielding a simulation box of $(58.5 \mu \mathrm{m} \times$ $88.4 \mu \mathrm{m} \times 106.8 \mu \mathrm{m})$. The code uses triangular-shaped clouds (TSC) [68], the Vay particle pusher [69], and the Esirkepov current deposition scheme [70]. Because of the high laser pulse intensities used in all simulations, which immediately and fully ionize hydrogen and helium in typical gas targets, all subsequent ionization dynamics were neglected by initializing the simulation with preionized plasma. The simulation duration is $5.0 \times 10^{5}$ time steps, corresponding to $41.9 \mathrm{ps}$. The laser pulses propagate in the $y z$ plane, and the electrons are accelerated in the $y$ direction. In order to avert unwanted interference of the spatially extended TWEAC laser pulses and the absorbing boundary layers surrounding the simulation volume, we apply a variant of the total-scattered field approach [71]. This technique implements a field-splitting scheme exploiting the linearity of Maxwell's equations in order to separate 
the incident TWEAC laser field from the scattered fields, i.e., the plasma response to the laser. As for minimizing unphysical reflections at the laser entrance and exit boundaries, the simulated plasma is terminated by linear plasma-vacuum transitions ( $1.53 \lambda_{0}$ thickness) before entering the absorbing boundary layers.

Both laser pulses are linearly polarized in $x$, propagate in the $y z$ plane, and feature 800-nm central wavelength, 10-fs (FWHM) transform-limited pulse duration, and a peak intensity of $2.62 \times 10^{19} \mathrm{~W} / \mathrm{cm}^{2}\left(a_{0}=3.5\right)$, respectively. Their propagation direction encloses an angle of $60^{\circ}$ and $-60^{\circ}$ with respect to the $y$ axis. The focal geometry is cylindrical, and the focal lines both coincide with the $y$ axis. The laser pulse focal line height is $w_{0, x}=1.2 \mu \mathrm{m}$. Since the focal line length $w_{0, y z}$ easily extends beyond the centimeter range, we assume, for the sake of simplicity, that the laser is unbounded in the $y z$ plane of the simulation. The pulsefront tilts are $\alpha= \pm 30^{\circ}$, respectively.

In our TWEAC scenarios, injection of a high charge electron bunch is done via shock injection [44-47]. First, the electron density $n_{e}$ linearly rises from vacuum to $6.4 \times 10^{18} \mathrm{~cm}^{-3}$ within a short distance of $20 \mu \mathrm{m}$. After this initial shock, $n_{e}$ linearly decreases on a longer scale, first to $4.5 \times 10^{18} \mathrm{~cm}^{-3}$ and finally to $3.2 \times 10^{18} \mathrm{~cm}^{-3}$ over subsequent distances of $160 \mu \mathrm{m}$ and $460 \mu \mathrm{m}$. This density configuration generates an initially high electron density at the back of the plasma cavity, which, due to the subsequent increase in plasma wavelength, $\lambda_{p} \propto n_{e}^{-1 / 2}$, and hence the surrounding plasma structure is injected into the accelerating volume of an expanded wakefield.

The reference laser-wakefield acceleration scenario is based on self-injection in a homogeneous plasma of $3.2 \times 10^{18} \mathrm{~cm}^{-3}$ in electron density (same as in TWEAC). The laser is a standard Gaussian laser pulse [800 nm, $30 \mathrm{fs}$ (FWHM), $w_{0}=7.9 \mu \mathrm{m} 1 / e^{2}$-spot size], focused to the beginning of the plasma. For improved comparability, the LWFA laser focal spot size is chosen $\left(k_{p} w_{0}=3.5\right)$ to result in a similar cavity diameter as the corresponding TWEAC cavity height along $x$. The intensity $1.05 \times 10^{20} \mathrm{~W} / \mathrm{cm}^{2}$ $\left(a_{0}=7.0\right)$ equals the maximum intensity of both TWEAC laser pulses at their crossing point. By selecting a scenario, where the dephasing length $L_{\mathrm{d}}=1.16 \times L_{\text {dep }}$ slightly exceeds the depletion length, one ensures that the LWFA laser pulse energy is fully exploited for acceleration before $L_{\mathrm{d}}$ is reached.

\section{Numerical challenges}

In contrast to LWFA, TWEAC simulations face unique numerical challenges: TWEAC simulations are not limited by the depletion and dephasing length and thus have a longer duration in terms of the plasma evolution than standard LWFA simulations of the same density. Furthermore, the two obliquely incident laser beams require high spatial grid resolution, not only in the longitudinal dimension as LWFA but also in at least one transverse dimension. Since the TWEAC geometry can neither be reduced in dimensions nor simplified by axial symmetry, it requires full 3D simulations. Advanced Lorentz-boosted frame-type solvers [72], which greatly speed up extended, multi-GeV LWFA simulations, cease to have an advantage in scenarios comprising two noncollinear laser beams. Beyond the acceleration lengths simulated in this work, numerical instabilities from the PIC solvers-such as from numerical dispersion, Cherenkov instabilities, spurious forces [73], and missing energy conservation in explicit solvers [74]-become an increasing challenge, leading to unphysical degradations in the accelerated electron beam quality. For TWEAC, alternative solvers that change the numerical speed of light around the common direction of both electrons and lasers [73] cannot be directly applied due to the laser beams propagating in two different, nonaxial direction. Advanced PIC methods, such as spectral solvers $[75,76]$, are expected to improve long-term numerical stability in future studies. For reducing the extended simulation time to solution required, a strategy could be to exploit the quasistationary acceleration conditions in reduced-physics simulations, such as quasistatic approximation [77] approaches.

\section{APPENDIX C: PERFORMANCE METRICS OF SMALL-ANGLE TWEAC SCENARIOS}

In Fig. 5(b), we adopted the characteristic wakefielddriver energy $\left[\eta_{\text {wake }}=W_{\text {laser }} / L_{\text {dep }}\right.$, Eqs. (2) and (4)], the laser pulse energy expended to sustain an accelerating plasma cavity over one LWFA depletion length, in order to compare the laser pulse requirements of LWFA and TWEAC and its general scaling for very different regimes.

Note that this quantity $\eta_{\text {wake }}$ excludes all details from the initial injection dynamics and charge; it also neglects LWFA interstage beam transport and LWFA gas target preparation from heater lasers. The characteristic wakefield-driver energy addresses the question of how much laser energy TWEAC fundamentally requires compared to LWFA to sustain a wakefield. Hence, the characteristic wakefield-driver energy denotes a useful first estimate to determine the possible acceleration efficiency.

In contrast to the LWFA acceleration gradient evolving from high gradients at injection until dephasing, the TWEAC acceleration gradients in this work are strongly determined by the initial acceleration gradient at injection. In this way, $\eta_{\text {wake }}$ helps us to separate detailed optimization of density profiles from the fundamental ability to sustain a wakefield for electron acceleration.

However, there are more efficiency indicators for describing laser-plasma accelerator performance. In Table I, we provide an overview over the simulations including injected charge, electron bunch size, acceleration gradient, characteristic wakefield-driver energy, acceleration volume of the first bucket, energy-gain efficiency, and laser-electron beam-energy efficiency. 
The energy-gain efficiency metric of laser pulse energy divided by the average electron energy gain describes the acceleration performance of a typical electron including the injection dynamics. However, this metric neglects the injected amount of charge and can be favorable even when only a few electrons are accelerated.

Thus, the laser-electron beam-energy efficiency, defined by the total kinetic electron beam energy divided by the total laser pulse energy, measures the degree of laser pulse energy exploitation. Typical LWFA values range from several percent up to the $20 \%$ range [17]. Depending on the application, less energy-efficient scenarios are used, when other beam parameters, such as charge, high peak current, low energy spread, or low emittance, are important optimization goals.

Although the injected charge depends primarily on the injection mechanism and can change drastically within the same accelerator regime, the volume of the accelerator cavity determines how much charge can be trapped and accelerated. For LWFA, on the one hand, this volume is associated with the optimal beam-loading limit [58-60] and mainly depends on laser intensity, laser spot size, and the electron plasma density. For the scenarios at $n_{e}=3.2 \times$ $10^{18} \mathrm{~cm}^{-3}$ presented here, one expects a charge in the tens of $\mathrm{pC}$ range $\left(Q_{\mathrm{opt}}=44 \mathrm{pC}\right)$. For the TWEAC geometry, on the other hand, the plasma cavity volume additionally depends both on the incidence angle $\phi$ and on the transverse gas target geometry. While for $\phi=60^{\circ}$, only a fraction of the laser beam is used, the plasma-cavity size is already much larger than the corresponding LWFA volume.

The $5^{\circ}$ scenario is comparable to LWFA with respect to the characteristic wakefield-driver energy and the acceleration. However, the detailed performance with respect to injected charge, energy-gain efficiency, and laser-electron beam-energy efficiency differs considerably. While the average acceleration gradient is higher for the LWFA scenario before reaching the dephasing and depletion limit, the TWEAC scenario at $5^{\circ}$ features better performance characteristics with respect to injected charge, energy-gain efficiency, and laser-electron beam-energy efficiency.

When going to lower angles at $\phi=3.5^{\circ}$, this trend to improve efficiency figures continues. Initial simulation tests have shown that with the same laser parameters and the same density profile, the injected amount of charge would exceed the beam-loading capacity of the plasma cavity. For this reason, we reduce the injected charge by lowering the peak laser strength from $a_{0}=3.5$ to 3.0 in each arm, reducing the plasma electron density from $n_{e}=$ $3.2 \times 10^{18} \mathrm{~cm}^{-3}$ to $2.5 \times 10^{18} \mathrm{~cm}^{-3}$, and employing a smoother gas jet profile with an initial density up-ramp of $120 \mu \mathrm{m}$ from vacuum to $n_{e}=5.0 \times 10^{18} \mathrm{~cm}^{-3}$, a plateau of $240 \mu \mathrm{m}$, and a shock transition to the final density $n_{e}=2.5 \times 10^{18} \mathrm{~cm}^{-3}$. With these adjustments, we conduct a 3D-PIConGPU simulation with a simulation box of $74.4 \mu \mathrm{m} \times 88.4 \mu \mathrm{m} \times 235.7 \mu \mathrm{m}$ and respective grid resolutions of $\left(\lambda_{0} / 12.0\right) \times\left(\lambda_{0} / 20.9\right) \times\left(\lambda_{0} / 20.9\right)$. The results not only show a higher acceleration gradient of $1.0 \mathrm{GeV} / \mathrm{cm}$ compared to the $5.0^{\circ}$ TWEAC scenario but feature excellent efficiency characteristics for all listed metrics. In particular, the laser-electron beam-energy efficiency, which reaches $47 \%$, is beyond the typical energy efficiency of about $20 \%$ for optimized LWFA scenarios [17]. This result is a direct consequence of the increased cavity size of about $60 \mu \mathrm{m}$ along the $z$ direction, which leads to improved utilization of the available laser pulse energy into the wake. This evidence shows the ability of TWEAC to accelerate with high energy efficiency.

Typical electron bunch dimensions during acceleration are $2.2 \mu \mathrm{m} \times 4.8 \mu \mathrm{m} \times 13 \mu \mathrm{m}(\mathrm{rms})$, indicating that flat beams could become possible in low-angle scenarios. Such flat beams are of great interest for collider applications in order to reduce beamstrahlung $[16,78]$.

With regard to applications, note that the injected charge of $1.77 \mathrm{nC}$ is beyond optimal LWFA beam-loading limits at tens of pC [58-60], so the observed energy spread [18.9\% (rms) at $330 \mathrm{MeV}]$ is significant. Although such accelerators can be highly desirable in applications where low energy spread is not a priority, such as for betatron radiation sources or for driving a subsequent plasma-wakefield stage $[79,80]$, many applications will require lower energy spreads. For such cases, the injection process should be tuned towards less injected charge by changing the density profile or by using alternative methods of injection, such as optical injection $[81,82]$.

In summary, for optimally exploiting the available laser energy in TWEAC, it is necessary to select a scenario in which the field-cycling length $L_{\text {cyc }}=L_{\text {trans }} / \sin \phi$ is a significant fraction of $L_{\mathrm{dep}}$. This objective can be achieved via shallow incidence angles $\phi$, high electron densities $n_{e}$, or short laser pulses to decrease $L_{\text {dep }}$, as well as larger laser foci to transversally extend the interaction zone $L_{\text {trans }}$ by longer focal lengths $Z_{0}$. The latter option is especially useful when aiming at flat electron beams for collider applications (see Fig. 7). However, as for LWFA, fully depleted TWEAC laser beams, $L_{\mathrm{cyc}}=L_{\mathrm{dep}}$, can negatively impact local cavity stability and thus electron beam quality.

\section{APPENDIX D: LIMITS TO THE CYCLING DISTANCE $L_{\text {cyc }}$}

Here, we present some additional information on how to determine the amount of laser energy depletion and thus possible limits of the characteristic propagation distance $L_{\text {cyc }}$ of each TWEAC laser to the focal line, where the main interaction occurs.

TWEAC essentially provides for a continuously refreshed laser field loaded from the side into the plasma channel. Only in the focal region does this field drive a plasma cavity, before being diffracted and replaced by another unperturbed field. TWEAC thus provides close to 


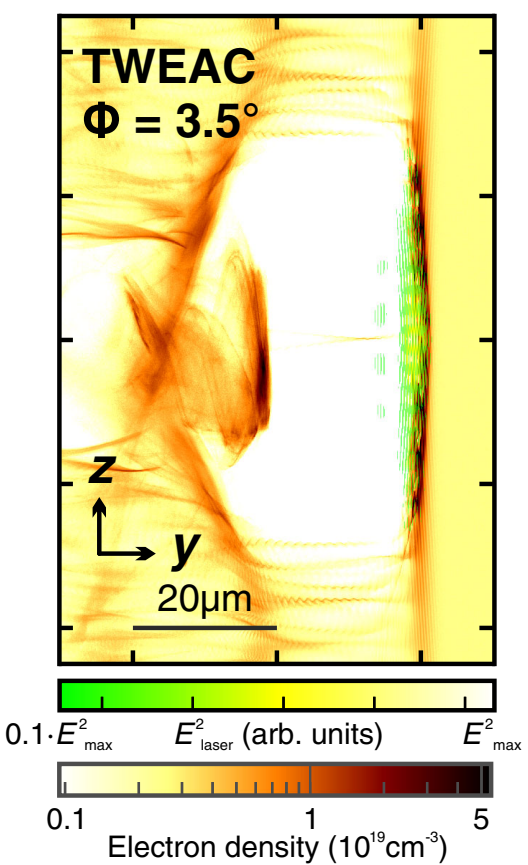

FIG. 7. The TWEAC cavity after shock injection using orthogonally polarized laser pulses at $3.5^{\circ}$ incidence angle. Compared to the $5^{\circ}$-TWEAC case, this scenario was optimized to reduce the injected charge while improving energy efficiency (see Table I), with laser-electron beam-energy efficiency reaching $47 \%$. The injected 1.8-nC electron bunch features dimensions of $2.2 \mu \mathrm{m} \times 4.8 \mu \mathrm{m} \times 13 \mu \mathrm{m}(\mathrm{rms})$.

perfect acceleration conditions similar to an unperturbed LWFA scenario and, unlike LWFA, retains these conditions over the complete acceleration length defined by the spatial extent of the TWEAC pulse projected onto the electron path of propagation.

Laser pump depletion from the laser pulse propagation to the interaction zone in the line focus is an energy cost offset that applies uniformly to the entire laser pulse front. If one section of the laser reaches the overlap with the second laser and drives a wakefield, the other TWEAC laser sections of the same intensity and pulse duration will also drive a wakefield-independent of the laser pulse width, so TWEAC stages can be arbitrarily long. If, however, the entire laser pulse is depleted by an extended initial propagation length through a dense plasma, the pulse front cannot drive a wakefield; thus, it prevents a TWEAC from the beginning. According to Eq. (4) and $L_{\mathrm{cyc}}>L_{\mathrm{dep}}$, this happens if the width of a slit-nozzle gas target-not its length - is too large, the incident angle $\phi$ is too shallow, the Rayleigh length of the cylindrical focus is too long, or the laser pulse duration is too short. Especially for experimental implementations aiming for maximum energy efficiency, it is critical to quantitatively study these limits.

Although laser energy depletion of LWFA is reasonably well understood $[15,83,84]$, the laser propagation of a cylindrically focused, obliquely incident TWEAC laser pulse in plasma, which, for most of its propagation distance, is not matched in height to the plasma wavelength, has not been studied yet in any detail. In the following, we present analytical estimates and contrast these with results from a 3D particle-in-cell simulation.

As an instructive example, we choose the $5^{\circ}$ scenario in order to demonstrate that a TWEAC of similar laser energy efficiency compared to LWFA remains energy efficient when it is experimentally implemented using a gas target width of $1 \mathrm{~mm}$. If longer laser pulses, lower electron densities, or more-narrow gas targets are used compared to such a worst-case reference scenario, one can expect less initial laser pump depletion, thus leaving more room for laser energy optimizations in TWEAC designs.

As a first analytical estimate, the etching speed according to $[15,84]$

$$
v_{\text {etch }}=c\left(\frac{\omega_{p}}{\omega_{0}}\right)^{2} \frac{\left(1+a_{0}^{2} / 2\right)^{2}-1}{\left(1+a_{0}^{2} / 2\right)^{2}}
$$

is useful for calculating the energy loss by depletion of a laser pulse with evolving laser intensity in a plasma of varying electron density since it includes the full $a_{0}$ dependence. Note that for $a_{0}<2$, the etching speed $v_{\text {etch }}$ scales proportionally to $a_{0}^{-2}$, which favors strong focusing for TWEAC and eases requirements to confine gas targets transversally to below $1 \mathrm{~mm}$.

Written in integral notation, the depleted energy fraction of the total laser pulse energy $W_{\text {laser }}$ becomes

$$
\begin{aligned}
\frac{\Delta W_{\text {dep }}}{W_{\text {laser }}} & =\frac{1}{c \tau_{0}} \int_{s_{0}}^{s_{1}} \frac{v_{\text {etch }}(s)}{c} \mathrm{~d} s \\
& =\frac{1}{c \tau_{0}} \int_{s_{0}}^{s_{1}}\left(\frac{\omega_{p}(s)}{\omega_{0}}\right)^{2} \frac{\left(1+a_{0}^{2}(s) / 2\right)^{2}-1}{\left(1+a_{0}^{2}(s) / 2\right)^{2}} \mathrm{~d} s
\end{aligned}
$$

with the plasma frequency $\omega_{p}(s)$ and the laser field strength $a_{0}(s)$ being functions of the laser propagation distance $s$. This depletion process only occurs if there exists a net momentum transfer between the laser and plasma by ponderomotively driving a wakefield. Thus, we define the integration interval $\left[s_{0}, s_{1}\right]$ as the section of the propagation distance in which the laser pulse height $w_{x}(s) \leq w_{x \text {, match }}(s)$ is equal to or smaller than the transverse matching conditions $[9,15]$ for driving a laser wakefield,

$$
\begin{aligned}
& w_{x, \text { match }}(s) \equiv \frac{2 c \sqrt{a_{0}(s)}}{\omega_{p}(s)}, \\
& w_{0, x} \cdot \sqrt{1+\left(\frac{s \cdot \sin \phi}{z_{R, x}}\right)^{2}}=\frac{2 c \sqrt{a_{0}(s)}}{\omega_{p}(s)}, \\
& \text { with } z_{R, x}=\pi w_{0, x}^{2} / \lambda_{0} .
\end{aligned}
$$


However, we emphasize that Eq. (D2) is only a first order-of-magnitude estimate for TWEAC lasers, which accounts neither for the very specific laser pulse and focusing geometry of TWEAC nor for different depletion dynamics of nonmatched or "overmatched" laser pulse heights. Hence, in order to compare with Eq. (D2), we also perform a 3D particle-in-cell simulation.

In contrast to LWFA, TWEAC laser pulses are obliquely incident within a cylindrical focal geometry, so for small incident angles $\phi=5^{\circ}$, the laser remains close to the focal region at high intensity and small laser beam height for an extended propagation distance proportional to $(\sin \phi)^{-1}$. Furthermore, the tight focus $w_{0, x}=1.2 \mu \mathrm{m}$ and extended propagation length of $5.7 \mathrm{~mm}$ break the paraxial approximation for ultrashort pulses in this scenario. Thus, we adopt the exact solution of the so-called "quasi-Gaussian cylindrical laser beam" in Ref. [85] [Eqs. (12)-(17)] and supplement the formalism with a laser envelope, which temporally defines the laser duration in the direction of propagation and spatially defines the laser beam width. The laser envelope follows the phase curvature, where the central, longitudinal slice at peak laser intensity follows a surface section of a cone with its main axis along the focal line and a half-opening angle of $(\pi / 2-\phi)$. The distance of the laser pulse to the focus is defined with respect to the position of the laser peak intensity.

Since the amount of laser energy depletion is not expected to correlate with the subsequent arrival times of different parts along the laser pulse front in the focus, for the sake of simplicity, we neglect, in this propagation simulation, the small $2.5^{\circ}$ pulse-front tilt of the $5^{\circ}$-TWEAC-type laser. Additionally, in order to calculate the amount of laser pump depletion for a single TWEAC-type laser pulse within a numerically tractable simulation volume and runtime, we constrain the laser pulse to a representative $50-\mu \mathrm{m}$ laser width, which is a fraction of the mm-to-cm pulse front of a full TWEAC-type laser. Here, we exploit the fact that every section of the laser pulse front has to traverse the same electron density profile from vacuum to the center of the gas target, where the TWEAC interaction takes place. The $50-\mu \mathrm{m}$ width is chosen to be large compared to the plasma wavelength of $18.7 \mu \mathrm{m}$ at $3.2 \times 10^{18} \mathrm{~cm}^{-3}$, as well as large compared to the corresponding Rayleigh length for preventing significant laser diffraction within the total propagation length to the center of the gas target.

As displayed in Fig. 8(a), the electron density profile is chosen to be $1 \mathrm{~mm}$ from vacuum to vacuum and thus corresponds to gas jets that are readily available in LWFA experiments. Since the laser pulse traverses the plasma at an oblique incidence angle of $5^{\circ}$, its effective propagation length to the center increases to $5.74 \mathrm{~mm}$ [see Fig. 8(c)]. Because of the cylindrical focus geometry along the $y$ axis, the laser will traverse the plasma at higher peak laser fields [Fig. 8(d)] compared to a laser where both transverse dimensions are being focused.
In a 3D-PIC simulation using PIConGPU, we initialize the $800-\mathrm{nm}, 10-\mathrm{fs}$ laser pulse with a peak intensity suitable for a subsequent laser strength of $a_{0}=3.5$ in a vacuum focus. The simulation volume $1469 \mu \mathrm{m} \times 113.4 \mu \mathrm{m} \times$ $1089 \mu \mathrm{m}$ features respective grid resolutions of $\left(\lambda_{0} / 1.1, \lambda_{0} / 9.0, \lambda_{0} / 1.1\right)$. According to the relative electron density profile of Fig. 8(c) with absolute peak electron density at $3.2 \times 10^{18} \mathrm{~cm}^{-3}$, we propagate the laser until it reaches the center of the gas jet.

The quantitative loss of field energy within the simulation volume is a measure of laser depletion. In order to gauge the difference between the field energy contained within the laser and the plasma fields, Fig. 8(b) displays both the total field energy from all electric and magnetic fields (blue line) and the $E_{x}$ field energy component (red line), corresponding to the linearly polarized laser in the $x$ direction, with respect to the propagation distance.

The resulting field energy loss of the $E_{x}$ field, which is close to the total loss in laser field energy, is $4.3 \%$ on the distance from vacuum to the laser focus. The corresponding analytical estimate Eq. (D2) using the electron density and the in-vacuum peak electric field along the propagation direction [Figs. 8(c) and 8(d)] predicts a laser energy loss of $12.8 \%$. Thus, the simulated result for the TWEAC laser is a third of this estimate.

In order to exhibit the mode quality of the focused laser, Fig. 9 depicts a $40 \mu \mathrm{m} \times 37 \mu \mathrm{m}$ zoom-out from the much larger $(1469 \mu \mathrm{m} \times 113.4 \mu \mathrm{m})$ simulation area. The 10-fs laser is tightly focused, drives a wakefield in the blowout regime, and reaches a peak laser strength of $a_{0}=3.35$, which is close to the intended $a_{0}=3.5$ and thus confirms the same trend seen in the energy field data in Fig. 8(b).

We note in passing that the simulation shows some selffocusing, shifting the laser focus away from the center of the gas jet. However, with this small level of depletion, such effects can be easily corrected in experiment by shifting the cylindrical mirror by approximately $37 \mu \mathrm{m}$ in the $z$ direction.

These results show that for TWEAC geometries, in contrast to standard LWFA scenarios, laser pump depletion can be minimized for ultrashort laser pulses in relatively dense plasmas of several $10^{18} \mathrm{~cm}^{-3}$ and long propagation lengths on the mm-to-cm scale as obtained from available experimental gas jets. In particular, this case is consistent with the scaling shown in Fig. 5(b), where both the minimum incidence angle of $0.2^{\circ}$ and laser pulse energy for this specific TWEAC scenario, according to the corresponding depletion limit condition $L_{\mathrm{cyc}}=L_{\mathrm{dep}}$, are more than an order of magnitude lower than the $5^{\circ}$ scenario examined here.

In part, this exceptionally small level of laser pump depletion can be explained according to the initial analytical estimate in Eqs. (D2) and (D3). On the one hand, a tightly focused cylindrical laser pulse spatially limits the laser propagation length at maximum intensity. On the 

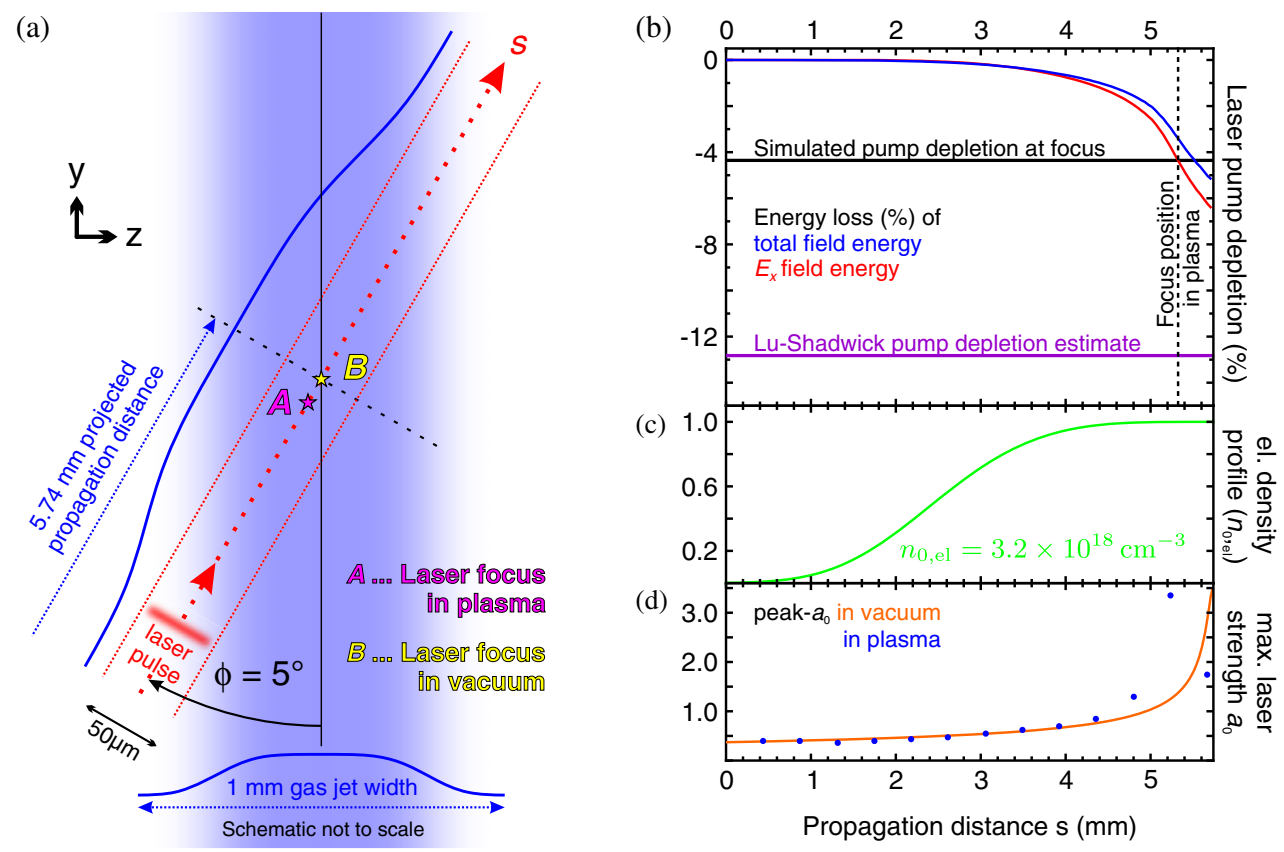

FIG. 8. (a) A schematic of the laser propagation setup. An 800-nm, 10-fs, linearly polarized laser with 50- $\mu$ m pulse-front width is cylindrically focused to $w_{0, x}=1.2-\mu \mathrm{m}$ focal line height and a maximum field strength of $a_{0}=3.5$ along the $y$ axis, which coincides with the center of a 1-mm-width slit-nozzle gas jet target. The laser pulse is obliquely incident at an angle of $5^{\circ}$. The $3 \mathrm{D}$-PIC simulation models the pulse evolution along 5.74-mm propagation distance from vacuum until the center of the gas jet. Marker $\boldsymbol{A}$ refers to the laser focus in plasma (spatial offset is due to self-focusing), while $\boldsymbol{B}$ denotes the original focus in vacuum. For better visibility, the schematic is not drawn to scale. (b) The relative loss of the total (blue line) and $E_{x}$ (red line) field energy in the simulation volume with respect to the propagation distance. The respective energy losses within the focus at $s=5.32 \mathrm{~mm}$ are $3.4 \%$ and $4.4 \%$, which is less than one-third of the analytical estimate of $12.8 \%$ derived from LWFA depletion according to Refs. [15,84]. (c) The assumed relative electron density profile projected along the laser direction of propagation. The super-Gaussian profile $n_{\mathrm{el}}(z)=3.2 \times 10^{18} \mathrm{~cm}^{-3} \cdot \exp \left(-z^{4} /\left(3.138 \times 10^{-4} \mathrm{~m}\right)^{4}\right)$ has an approximate vacuum-to-vacuum width of $1 \mathrm{~mm}$. However, the projected density profile seen by the laser is $n_{\mathrm{el}}(s \cdot \sin (\phi))$. (d) The laser pulse peak electric field in the dimensionless laser strength unit $a_{0}$ along the laser direction of propagation for in-vacuum propagation (solid) compared to the propagation in plasma (blue markers), where self-focusing dynamics leads to an earlier focus.

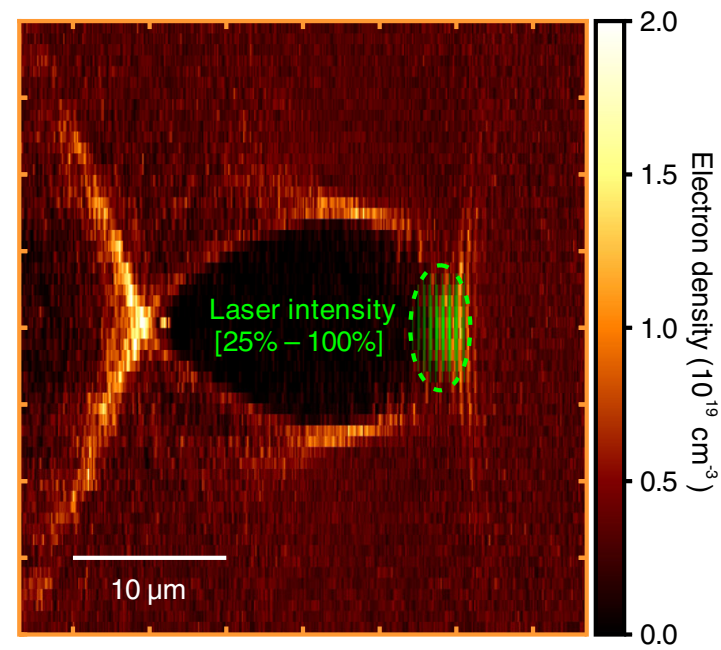

FIG. 9. A zoomed-in slice of the laser intensity (green) and electron density near the focus at $s=5.23 \mathrm{~mm}$. The tightly focused laser drives a wakefield and features a peak laser strength of $a_{0}=3.35$, which is close to the maximum $a_{0}=3.5$ at the vacuum line focus. The laser intensity scale (green) ranges from $25 \%$ to $100 \%$ relative peak intensity. other hand, a short depth of focus also limits suitable transverse matching conditions. These constraints avert driving a wakefield outside the cylindrical focus and thus preclude unwanted laser pump depletion of ultrashort laser pulses shorter than the plasma wavelength.

[1] T. Tajima and J. M. Dawson, Laser Electron-Accelerator, Phys. Rev. Lett. 43, 267 (1979).

[2] A. Pukhov and J. Meyer-ter Vehn, Laser Wake Field Acceleration: The Highly Non-linear Broken-Wave Regime, Appl. Phys. B 74, 355 (2002).

[3] S. Mangles, C. Murphy, Z. Najmudin, A. Thomas, J. Collier, A. Dangor, E. J. Divall, P. Foster, J. Gallacher, C. Hooker et al., Monoenergetic Beams of Relativistic Electrons from Intense Laser-Plasma Interactions, Nature (London) 431, 535 (2004).

[4] C. Geddes, C. Toth, J. Van Tilborg, E. Esarey, C. Schroeder, D. Bruhwiler, C. Nieter, J. Cary, and W. Leemans, HighQuality Electron Beams from a Laser Wakefield Accelerator 
Using Plasma-Channel Guiding, Nature (London) 431, 538 (2004).

[5] J. Faure, Y. Glinec, A. Pukhov, S. Kiselev, S. Gordienko, E. Lefebvre, J.-P. Rousseau, F. Burgy, and V. Malka, A LaserPlasma Accelerator Producing Monoenergetic Electron Beams, Nature (London) 431, 541 (2004).

[6] J. Faure, C. Rechatin, A. Norlin, A. Lifschitz, Y. Glinec, and V. Malka, Controlled Injection and Acceleration of Electrons in Plasma Wakefields by Colliding Laser Pulses, Nature (London) 444, 737 (2006).

[7] W. P. Leemans, B. Nagler, A. J. Gonsalves, C. Tóth, K. Nakamura, C. G. R. Geddes, E. Esarey, C. B. Schroeder, and S. M. Hooker, GeV Electron Beams from a CentimetreScale Accelerator, Nat. Phys. 2, 696 (2006).

[8] J. Osterhoff, A. Popp, Z. Major, B. Marx, T. P. RowlandsRees, M. Fuchs, M. Geissler, R. Hörlein, B. Hidding, S. Becker, E. A. Peralta, U. Schramm, F. Grüner, D. Habs, F. Krausz, S. M. Hooker, and S. Karsch, Generation of Stable, Low-Divergence Electron Beams by Laser-Wakefield Acceleration in a Steady-State-Flow Gas Cell, Phys. Rev. Lett. 101, 085002 (2008).

[9] E. Esarey, C. B. Schroeder, and W. P. Leemans, Physics of Laser-Driven Plasma-Based Electron Accelerators, Rev. Mod. Phys. 81, 1229 (2009).

[10] X. Wang et al., Quasi-monoenergetic Laser-Plasma Acceleration of Electrons to $2 \mathrm{GeV}$, Nat. Commun. 4, 1988 (2013).

[11] W. P. Leemans, A. J. Gonsalves, H.-S. Mao, K. Nakamura, C. Benedetti, C. B. Schroeder, C. Tóth, J. Daniels, D. E. Mittelberger, S. S. Bulanov, J.-L. Vay, C. G. R. Geddes, and E. Esarey, Multi-GeV Electron Beams from CapillaryDischarge-Guided Subpetawatt Laser Pulses in the SelfTrapping Regime, Phys. Rev. Lett. 113, 245002 (2014).

[12] W. T. Wang, W. T. Li, J. S. Liu, Z. J. Zhang, R. Qi, C. H. Yu, J. Q. Liu, M. Fang, Z. Y. Qin, C. Wang, Y. Xu, F. X. Wu, Y. X. Leng, R. X. Li, and Z. Z. Xu, High-Brightness HighEnergy Electron Beams from a Laser Wakefield Accelerator via Energy Chirp Control, Phys. Rev. Lett. 117, 124801 (2016).

[13] A. J. Gonsalves et al., Petawatt Laser Guiding and Electron Beam Acceleration to $8 \mathrm{GeV}$ in a Laser-Heated Capillary Discharge Waveguide, Phys. Rev. Lett. 122, 084801 (2019).

[14] V. Malka, S. Fritzler, E. Lefebvre, M. M. Aleonard, F. Burgy, J. P. Chambaret, J. F. Chemin, K. Krushelnick, G. Malka, S. P. Mangles, Z. Najmudin, M. Pittman, J. P. Rousseau, J. N. Scheurer, B. Walton, and A. E. Dangor, Electron Acceleration by a Wake Field Forced by an Intense Ultrashort Laser Pulse, Science 298, 1596 (2002).

[15] W. Lu, M. Tzoufras, C. Joshi, F. Tsung, W. Mori, J. Vieira, R. Fonseca, and L. Silva, Generating Multi-GeV Electron Bunches Using Single Stage Laser Wakefield Acceleration in a 3D Nonlinear Regime, Phys. Rev. ST Accel. Beams 10, 061301 (2007).

[16] C. B. Schroeder, E. Esarey, and W. P. Leemans, Beamstrahlung Considerations in Laser-Plasma-Accelerator-Based Linear Colliders, Phys. Rev. ST Accel. Beams 15, 051301 (2012).

[17] C. B. Schroeder, E. Esarey, C. G. R. Geddes, C. Benedetti, and W. P. Leemans, Physics Considerations for Laser-
Plasma Linear Colliders, Phys. Rev. ST Accel. Beams 13, 101301 (2010).

[18] S. P. D. Mangles, G. Genoud, M. S. Bloom, M. Burza, Z. Najmudin, A. Persson, K. Svensson, A. G. R. Thomas, and C. G. Wahlström, Self-Injection Threshold in Self-Guided Laser Wakefield Accelerators, Phys. Rev. ST Accel. Beams 15, 011302 (2012).

[19] S. Steinke, J. van Tilborg, C. Benedetti, C. G. R. Geddes, C. B. Schroeder, J. Daniels, K. K. Swanson, A. J. Gonsalves, K. Nakamura, N. H. Matlis, B. H. Shaw, E. Esarey, and W. P. Leemans, Multistage Coupling of Independent LaserPlasma Accelerators, Nature (London) 530, 190 (2016).

[20] I. Dornmair, K. Floettmann, and A. R. Maier, Emittance Conservation by Tailored Focusing Profiles in a Plasma Accelerator, Phys. Rev. ST Accel. Beams 18, 041302 (2015).

[21] M. Migliorati, A. Bacci, C. Benedetti, E. Chiadroni, M. Ferrario, A. Mostacci, L. Palumbo, A. R. Rossi, L. Serafini, and P. Antici, Intrinsic Normalized Emittance Growth in Laser-Driven Electron Accelerators, Phys. Rev. ST Accel. Beams 16, 011302 (2013).

[22] T. Mehrling, J. Grebenyuk, F. S. Tsung, K. Floettmann, and J. Osterhoff, Transverse Emittance Growth in Staged LaserWakefield Acceleration, Phys. Rev. ST Accel. Beams 15, 111303 (2012).

[23] P. Antici, A. Bacci, C. Benedetti, E. Chiadroni, M. Ferrario, A. R. Rossi, L. Lancia, M. Migliorati, A. Mostacci, L. Palumbo, and L. Serafini, Laser-Driven Electron Beamlines Generated by Coupling Laser-Plasma Sources with Conventional Transport Systems, J. Appl. Phys. 112, 044902 (2012).

[24] S. V. Bulanov, T. Z. Esirkepov, Y. Hayashi, H. Kiriyama, J. Koga, H. Kotaki, M. Mori, and M. Kando, On Some Theoretical Problems of Laser Wake-Field Accelerators, J. Plasma Phys. 82, 905820308 (2016).

[25] S. V. Bulanov, V. A. Vshivkov, G. I. Dudnikova, N. M. Naumova, F. Pegoraro, and I. V. Pogorelsky, Laser Acceleration of Charged Particles in Inhomogeneous Plasmas. I, Plasma Phys. Rep. 23, 259 (1997).

[26] E. Guillaume, A. Döpp, C. Thaury, K. T. Phuoc, A. Lifschitz, G. Grittani, J.-P. Goddet, A. Tafzi, S. W. Chou, L. Veisz, and V. Malka, Electron Rephasing in a LaserWakefield Accelerator, Phys. Rev. Lett. 115, 155002 (2015).

[27] P. Sprangle, B. Hafizi, J. R. Peñano, R. F. Hubbard, A. Ting, C. I. Moore, D. F. Gordon, A. Zigler, D. Kaganovich, and T. M. Antonsen, Wakefield Generation and GeV Acceleration in Tapered Plasma Channels, Phys. Rev. E 63, 056405 (2001).

[28] E. Gschwendtner et al., AWAKE, The Advanced Proton Driven Plasma Wakefield Acceleration Experiment at CERN, Nucl. Instrum. Methods Phys. Res., Sect. A 829, 76 (2016).

[29] M. Litos, E. Adli et al., High-Efficiency Acceleration of an Electron Beam in a Plasma Wakefield Accelerator, Nature (London) 515, 92 (2014).

[30] M. J. Hogan, T. O. Raubenheimer, A. Seryi, P. Muggli, T. Katsouleas, C. Huang, W. Lu, W. An, K. A. Marsh, W. B. Mori, C. E. Clayton, and C. Joshi, Plasma Wakefield Acceleration Experiments at FACET, New J. Phys. 12, 055030 (2010). 
[31] I. Blumenfeld, C. E. Clayton, F.-J. Decker, M. J. Hogan, C. Huang, R. Ischebeck, R. Iverson, C. Joshi, T. Katsouleas, N. Kirby, W. Lu, K. A. Marsh, W. B. Mori, P. Muggli, E. Oz, R. H. Siemann, D. Walz, and M. Zhou, Energy Doubling of $42 \mathrm{GeV}$ Electrons in a Metre-Scale Plasma Wakefield Accelerator, Nature (London) 445, 741 (2007).

[32] A. Caldwell, K. Lotov, A. Pukhov, and F. Simon, ProtonDriven Plasma-Wakefield Acceleration, Nat. Phys. 5, 363 (2009).

[33] K. Nakajima, Laser Electron Acceleration Beyond $100 \mathrm{GeV}$, Eur. Phys. J. Spec. Top. 223, 999 (2014).

[34] K. Nakajima, A. Deng, X. Zhang, B. Shen, J. Liu, R. Li, Z. Xu, T. Ostermayr, S. Petrovics, C. Klier, K. Iqbal, H. Ruhl, and T. Tajima, Operating Plasma Density Issues on LargeScale Laser-Plasma Accelerators Toward High-Energy Frontier, Phys. Rev. ST Accel. Beams 14, 091301 (2011).

[35] W. Leemans and E. Esarey, Laser-Driven Plasma-Wave Electron Accelerators, Phys. Today 62, No. 3, 44 (2009).

[36] I. Hinchliffe and M. Battaglia, A TeV Linear Collider, Phys. Today 57, No. 9, 49 (2004).

[37] J. Ellis and I. Wilson, New Physics with the Compact Linear Collider, Nature (London) 409, 431 (2001).

[38] K. Steiniger, M. Bussmann, R. Pausch, T. Cowan, A. Irman, A. Jochmann, R. Sauerbrey, U. Schramm, and A. Debus, Optical Free-Electron Lasers with Traveling-Wave Thomson-Scattering, J. Phys. B 47, 234011 (2014).

[39] A. D. Debus, M. Bussmann, M. Siebold, A. Jochmann, U. Schramm, T. E. Cowan, and R. Sauerbrey, Traveling-Wave Thomson Scattering and Optical Undulators for High-Yield EUV and X-ray Sources, Appl. Phys. B 100, 61 (2010).

[40] K. Steiniger, R. Widera, R. Pausch, A. Debus, M. Bussmann, and U. Schramm, Wave Optical Description of the TravelingWave Thomson-Scattering Optical Undulator Field and Its Application to the TWTS-FEL, Nucl. Instrum. Methods Phys. Res., Sect. A 740, 147 (2014).

[41] K. Steiniger, A. Debus, A. Irman, A. Jochmann, R. Pausch, U. Schramm, T. Cowan, and M. Bussmann, All-Optical Free-Electron Lasers Using Traveling-Wave ThomsonScattering, in IPAC 2014: Proceedings of the 5th International Particle Accelerator Conference, Dresden, Germany, (JACoW, Geneva, Switzerland, 2014).

[42] K. Steiniger, D. Albach, M. Bussmann, M. Loeser, R. Pausch, F. Röser, U. Schramm, M. Siebold, and A. Debus, Building an Optical Free-Electron Laser in the TravelingWave Thomson-Scattering Geometry, Front. Phys. 6, 155 (2019).

[43] PIConGPU-A Many-GPGPU Particle-in-Cell Code, http:// picongpu.hzdr.de.

[44] A. Buck, J. Wenz, J. Xu, K. Khrennikov, K. Schmid, M. Heigoldt, J. M. Mikhailova, M. Geissler, B. Shen, F. Krausz, S. Karsch, and L. Veisz, Shock-Front Injector for HighQuality Laser-Plasma Acceleration, Phys. Rev. Lett. 110, 185006 (2013).

[45] A. J. Gonsalves, K. Nakamura, C. Lin, D. Panasenko, S. Shiraishi, T. Sokollik, C. Benedetti, C. B. Schroeder, C. G. R. Geddes, J. van Tilborg, J. Osterhoff, E. Esarey, C. Toth, and W. P. Leemans, Tunable Laser Plasma Accelerator Based on Longitudinal Density Tailoring, Nat. Phys. 7, 862 (2011).

[46] K. Schmid, A. Buck, C. M. S. Sears, J. M. Mikhailova, R. Tautz, D. Herrmann, M. Geissler, F. Krausz, and L. Veisz,
Density-Transition Based Electron Injector for Laser Driven Wakefield Accelerators, Phys. Rev. ST Accel. Beams 13, 091301 (2010).

[47] S. V. Bulanov, N. Naumova, F. Pegoraro, and J. Sakai, Particle Injection into the Wave Acceleration Phase Due to Nonlinear Wake Wave Breaking, Phys. Rev. E 58, R5257 (1998).

[48] P. Sprangle, C. M. Tang, and E. Esarey, Relativistic SelfFocusing of Short-Pulse Radiation Beams in Plasmas, IEEE Trans. Plasma Sci. 15, 145 (1987).

[49] A. E. Kaplan and A. L. Pokrovsky, Fully Relativistic Theory of the Ponderomotive Force in an Ultraintense Standing Wave, Phys. Rev. Lett. 95, 053601 (2005).

[50] A. L. Pokrovsky and A. E. Kaplan, Relativistic Reversal of the Ponderomotive Force in a Standing Laser Wave, Phys. Rev. A 72, 043401 (2005).

[51] E. N. Frolov, A. V. Dik, S. B. Dabagov, and K. P. Artyomov, On Channeling Potential for Relativistic Electrons in Crossed Laser Field, J. Phys. Conf. Ser. 552, 012035 (2014).

[52] Z. M. Sheng, K. Mima, J. Zhang, and J. Meyer-Ter-Vehn, Efficient Acceleration of Electrons with Counterpropagating Intense Laser Pulses in Vacuum and Underdense Plasma, Phys. Rev. E 69, 016407 (2004).

[53] P. Zhang, N. Saleh, S. Chen, Z. M. Sheng, and D. Umstadter, Laser-Energy Transfer and Enhancement of Plasma Waves and Electron Beams by Interfering High-Intensity Laser Pulses, Phys. Rev. Lett. 91, 225001 (2003).

[54] L.-L. Yu, E. Esarey, C. B. Schroeder, J.-L. Vay, C. Benedetti, C. G. R. Geddes, M. Chen, and W. P. Leemans, Two-Color Laser-Ionization Injection, Phys. Rev. Lett. 112, 125001 (2014).

[55] B. Hidding, J. B. Rosenzweig, Y. Xi, B. O'Shea, G. Andonian, D. Schiller, S. Barber, O. Williams, G. Pretzler, T. Königstein, F. Kleeschulte, M. J. Hogan, M. Litos, S. Corde, W. W. White, P. Muggli, D. L. Bruhwiler, and K. Lotov, Beyond Injection: Trojan Horse Underdense Photocathode Plasma Wakefield Acceleration, AIP Conf. Proc. 1507, 570 (2012).

[56] T. P. Wangler, RF Linear Accelerators, 2nd ed. (WileyVCH, Weinheim, Germany, 2008).

[57] R. B. R-Shersby-Harvie, Travelling Wave Linear Accelerators, Proc. Phys. Soc. 61, 255 (1948).

[58] M. Tzoufras, W. Lu, F. S. Tsung, C. Huang, W. B. Mori, T. Katsouleas, J. Vieira, R. A. Fonseca, and L. O. Silva, Beam Loading in the Nonlinear Regime of Plasma-Based Acceleration, Phys. Rev. Lett. 101, 145002 (2008).

[59] M. Tzoufras, W. Lu, F. S. Tsung, C. Huang, W. B. Mori, T. Katsouleas, J. Vieira, R. A. Fonseca, and L. O. Silva, Beam Loading by Electrons in Nonlinear Plasma Wakes, Phys. Plasmas 16, 056705 (2009).

[60] J. P. Couperus, R. Pausch, A. Köhler, O. Zarini, J. M. Krämer, M. Garten, A. Huebl, R. Gebhardt, U. Helbig, S. Bock, K. Zeil, A. Debus, M. Bussmann, U. Schramm, and A. Irman, Demonstration of a Beam Loaded NanocoulombClass Laser Wakefield Accelerator, Nat. Commun. 8, 487 (2017).

[61] S. Kühn et al., The ELI-ALPS Facility: The Next Generation of Attosecond Sources, J. Phys. B 50, 132002 (2017).

[62] A. J. Gonsalves, F. Liu, N. A. Bobrova, P. V. Sasorov, C. Pieronek, J. Daniels, S. Antipov, J. E. Butler, S. S. Bulanov, 
W. L. Waldron, D. E. Mittelberger, and W. P. Leemans, Demonstration of a High Repetition Rate Capillary Discharge Waveguide, J. Appl. Phys., 033302119 (2016).

[63] N. Zamfir, Extreme Light Infrastructure Nuclear Physics (ELI-NP): Present Status and Perspectives, EPJ Web Conf. 117, 10001 (2016).

[64] U. Schramm et al., First Results with the Novel Petawatt Laser Acceleration Facility in Dresden, J. Phys. Conf. Ser. 874, 012028 (2017).

[65] A. S. Pirozhkov, Y. Fukuda, M. Nishiuchi, H. Kiriyama, A. Sagisaka, K. Ogura, M. Mori, M. Kishimoto, H. Sakaki, N. P. Dover, K. Kondo, N. Nakanii, K. Huang, M. Kanasaki, K. Kondo, and M. Kando, Approaching the DiffractionLimited, Bandwidth-Limited Petawatt, Opt. Express 25, 20486 (2017).

[66] M. Bussmann, H. Burau, T. E. Cowan, A. Debus, A. Huebl, G. Juckeland, T. Kluge, W. E. Nagel, R. Pausch, F. Schmitt, U. Schramm, J. Schuchart, and R. Widera, Radiative Signatures of the Relativistic Kelvin-Helmholtz Instability, in Proceedings of the International Conference on High Performance Computing, Networking, Storage and Analysis, SC '13 (ACM, New York, NY, 2013), pp. 5:1-5:12, http://dx.doi.org/10.1145/2503210.2504564.

[67] A. Debus, K. Steiniger, R. Pausch, A. Huebl, and R. Widera, PIConGPU Simulation Settings for TWEAC, https://doi.org/ 10.14278/rodare.150 (2019).

[68] R. W. Hockney and J. W. Eastwood, Computer Simulation Using Particles (Taylor \& Francis, Bristol, PA, 1988).

[69] J.-L. Vay, D. P. Grote, R. H. Cohen, and A. Friedman, Novel Methods in the Particle-In-Cell Accelerator CodeFramework Warp, Comput. Sci. Discovery 5, 014019 (2012).

[70] T. Esirkepov, Exact Charge Conservation Scheme for Particle-in-Cell Simulation with an Arbitrary Form-Factor, Comput. Phys. Commun. 135, 144 (2001).

[71] A. Taflove and S. Hagness, Computational Electrodynamics: The Finite-Difference Time-Domain Method, 3rd ed., Artech House Antennas and Propagation Library (Artech House, Norwood, MA, 2005), Chap. 5.

[72] J.-L. Vay, Noninvariance of Space- and Time-Scale Ranges under a Lorentz Transformation and the Implications for the Study of Relativistic Interactions, Phys. Rev. Lett. 98, 130405 (2007).

[73] R. Lehe, A. Lifschitz, C. Thaury, V. Malka, and X. Davoine, Numerical Growth of Emittance in Simulations of LaserWakefield Acceleration, Phys. Rev. ST Accel. Beams 16, 021301 (2013).
[74] S. Markidis and G. Lapenta, The Energy Conserving Particle-in-Cell Method, J. Comput. Phys. 230, 7037 (2011).

[75] S. Jalas, I. Dornmair, R. Lehe, H. Vincenti, J.-L. Vay, M. Kirchen, and A.R. Maier, Accurate Modeling of Plasma Acceleration with Arbitrary Order Pseudo-spectral Particle-in-Cell Methods, Phys. Plasmas 24, 033115 (2017).

[76] J.-L. Vay, I. Haber, and B. B. Godfrey, A Domain Decomposition Method for Pseudo-spectral Electromagnetic Simulations of Plasmas, J. Comput. Phys. 243, 260 (2013).

[77] C. Huang, V. K. Decyk, M. Zhou, W. Lu, W. B. Mori, J. H. Cooley, T. M. Antonsen, Jr., B. Feng, T. Katsouleas, J. Vieira, and L. O. Silva, QuickPIC: A Highly Efficient Fully Parallelized PIC Code for Plasma-Based Acceleration, J. Phys. Conf. Ser. 46, 190 (2006).

[78] R. Brinkmann, Y. Derbenev, and K. Flöttmann, A Low Emittance, Flat-Beam Electron Source for Linear Colliders, Phys. Rev. ST Accel. Beams 4, 053501 (2001).

[79] B. Hidding, T. Königstein, J. Osterholz, S. Karsch, O. Willi, and G. Pretzler, Monoenergetic Energy Doubling in a Hybrid Laser-Plasma Wakefield Accelerator, Phys. Rev. Lett. 104, 195002 (2010).

[80] B. Hidding, G. G. Manahan, O. Karger, A. Knetsch, G. Wittig, D. A. Jaroszynski, Z. M. Sheng, Y. Xi, A. Deng, J. B. Rosenzweig, G. Andonian, A. Murokh, G. Pretzler, D. L. Bruhwiler, and J. Smith, Ultrahigh Brightness Bunches from Hybrid Plasma Accelerators as Drivers of 5th Generation Light Sources, J. Phys. B 47, 234010 (2014).

[81] C. Rechatin, J. Faure, A. Ben-Ismail, J. Lim, R. Fitour, A. Specka, H. Videau, A. Tafzi, F. Burgy, and V. Malka, Controlling the Phase-Space Volume of Injected Electrons in a Laser-Plasma Accelerator, Phys. Rev. Lett. 102, 164801 (2009).

[82] X. Davoine, E. Lefebvre, C. Rechatin, J. Faure, and V. Malka, Cold Optical Injection Producing Monoenergetic, Multi-GeV Electron Bunches, Phys. Rev. Lett. 102, 065001 (2009).

[83] C. D. Decker, W. B. Mori, K.-C. Tzeng, and T. Katsouleas, The Evolution of Ultra-intense, Short-Pulse Lasers in Underdense Plasmas, Phys. Plasmas 3, 2047 (1996).

[84] B. A. Shadwick, C. B. Schroeder, and E. Esarey, Nonlinear Laser Energy Depletion in Laser-Plasma Accelerators, Phys. Plasmas 16, 056704 (2009).

[85] F. G. Mitri, Cylindrical Quasi-Gaussian Beams, Opt. Lett. 38, 4727 (2013). 